\title{
Cosmic ray oriented performance studies for the JEM-EUSO first level trigger
}

G. Abdellaoui ${ }^{8}$, S. Abe ${ }^{54}$, A. Acheli ${ }^{1}$, J.H. Adams Jr. ${ }^{90}$, S. Ahmad ${ }^{11}$, A. Ahriche ${ }^{5}$, J.-N. Albert ${ }^{10}$, D. Allard ${ }^{12}$, G. Alonso ${ }^{81}$, L. Anchordoqui ${ }^{92}$, V. Andreev ${ }^{91}$, A. Anzalone ${ }^{25,31}$, W. Aouimeur ${ }^{1}$, Y. Arai ${ }^{56}$, N. Arsene ${ }^{72}$, K. Asano ${ }^{40}$, R. Attallah ${ }^{3}$, H. Attoui ${ }^{1}$, M. Ave Pernas ${ }^{80}$, S. Bacholle ${ }^{12}$, M. Bakiri ${ }^{1}$, P. Baragatti ${ }^{32}$, P. Barrillon ${ }^{10}$, S. Bartocci ${ }^{32}$, T. Batsch ${ }^{70}$, J. Bayer ${ }^{17}$, R. Bechini ${ }^{29}$, T. Belenguer ${ }^{79}$, R. Bellotti ${ }^{18,19}$, A. Belov ${ }^{75}$, K. Belov ${ }^{91}$, B. Benadda ${ }^{8}$, K. Benmessai ${ }^{7}$, A.A. Berlind ${ }^{94}$, M. Bertaina ${ }^{28,29, *}$, P.L. Biermann ${ }^{15}$, S. Biktemerova ${ }^{73}$, F. Bisconti ${ }^{15}$, N. Blanc ${ }^{84}$, J. Błęcki ${ }^{71}$, S. Blin-Bondil ${ }^{11}$, P. Bobik ${ }^{76}$, M. Bogomilov ${ }^{9}$, M. Bonamente ${ }^{90}$, R. Boudaoud ${ }^{1}$, E. Bozzo ${ }^{85}$, M.S. Briggs ${ }^{90}$, A. Bruno ${ }^{19}$, K.S. Caballero ${ }^{66}$, F. Cafagna ${ }^{18}$, D. Campana ${ }^{23}$, J.-N. Capdevielle ${ }^{12}$, F. Capel ${ }^{83}$, A. Caramete ${ }^{72}$, L. Caramete ${ }^{72}$, P. Carlson ${ }^{83}$, R. Caruso ${ }^{20,31, *}$, M. Casolino ${ }^{57,26}$, C. Cassardo ${ }^{28,29}$, A. Castellina ${ }^{28,30}$, G. Castellini ${ }^{21}$, C. Catalano ${ }^{13}$, O. Catalano ${ }^{25,31, *}$, A. Cellino ${ }^{28,30}$, M. Chikawa ${ }^{37}$, G. Chiritoi $^{72}$, M.J. Christl ${ }^{93}$, V. Connaughton ${ }^{90}$, L. Conti ${ }^{32}$, G. Contino ${ }^{20,31}$, G. Cordero ${ }^{62}$, G. Cotto ${ }^{28,29}$, H.J. Crawford ${ }^{87}$, R. Cremonini ${ }^{29}$, S. Csorna ${ }^{94}$, S. Dagoret-Campagne ${ }^{10}$, C. De Donato ${ }^{26}$, C. de la Taille ${ }^{11}$, C. De Santis ${ }^{26}$, L. del Peral ${ }^{80}$, M. Di Martino ${ }^{30}$, T. Djemil ${ }^{3}$, S.A. Djenas ${ }^{8}$, F. Dulucq ${ }^{11}$, M. Dupieux ${ }^{13}$, I. Dutan ${ }^{72}$, A. Ebersoldt ${ }^{15}$, T. Ebisuzaki ${ }^{57}$, R. Engel $^{15}$, J. Eser ${ }^{89}$, K. Fang ${ }^{88}$, F. Fenu ${ }^{28,29}$, S. Fernández-González ${ }^{78}$, J. Fernández-Soriano ${ }^{80}$, S. Ferrarese ${ }^{28,29}$, D. Finco $^{32}$, M. Flamini ${ }^{32}$, C. Fornaro ${ }^{32}$, R. Forza ${ }^{28,29}$, M. Fouka ${ }^{2}$, A. Franceschi ${ }^{22}$, S. Franchini ${ }^{81}$, C. Fuglesang ${ }^{83}$, J. Fujimoto ${ }^{56}$, M. Fukushima ${ }^{40}$, P. Galeotti ${ }^{28,29}$, E. García-Ortega ${ }^{78}$, G. Garipov ${ }^{75}$, E. Gascón ${ }^{78}$, J. Geary ${ }^{90}$, G. Gelmini ${ }^{91}$, J. Genci ${ }^{77}$, G. Giraudo ${ }^{28}$, M. Gonchar ${ }^{73}$, C. González Alvarado ${ }^{79}$, P. Gorodetzky ${ }^{12}$, N. Guardone ${ }^{20,31}$, F. Guarino ${ }^{23,24}$, R. Guehaz ${ }^{1}$, A. Guzmán ${ }^{17}$, Y. Hachisu ${ }^{57}$, M. Haiduc ${ }^{72}$, B. Harlov ${ }^{74}$, A. Haungs ${ }^{15}$, J. Hernández Carretero ${ }^{80}$, W. Hidber ${ }^{62}$, K. Higashide ${ }^{51,57}$, D. Ikeda ${ }^{40}$, H. Ikeda ${ }^{49}$, N. Inoue ${ }^{51}$, S. Inoue ${ }^{57}$, A. Insolia ${ }^{20,31}$, F. Isgrò ${ }^{23,33}$, Y. Itow ${ }^{47}$, T. Jammer ${ }^{16}$, E. Joven ${ }^{82}$, E.G. Judd ${ }^{87}$, A. Jung ${ }^{12}$, J. Jochum ${ }^{16}$, F. Kajino ${ }^{42}$, T. Kajino ${ }^{45}$, S. Kalli ${ }^{6}$, I. Kaneko ${ }^{57}$, D. Kang ${ }^{15}$, F. Kanouni ${ }^{7}$, Y. Karadzhov ${ }^{9}$, J. Karczmarczyk ${ }^{70}$, M. Karus ${ }^{15}$, K. Katahira ${ }^{57}$, K. Kawai ${ }^{57}$, Y. Kawasaki ${ }^{57}$, A. Kedadra ${ }^{1}$, H. Khales ${ }^{1}$, B.A. Khrenov ${ }^{75}$, Jeong-Sook Kim ${ }^{58}$, Soon-Wook Kim ${ }^{58}$, Sug-Whan Kim ${ }^{61}$, M. Kleifges ${ }^{15}$, P.A. Klimov ${ }^{75}$, D. Kolev ${ }^{9}$,

I. Kreykenbohm ${ }^{14}$, K. Kudela ${ }^{76}$, Y. Kurihara ${ }^{56}$, A. Kusenko ${ }^{55,91}$, E. Kuznetsov ${ }^{90}$, M. Lacombe ${ }^{13}$, C. Lachaud $^{12}$, H. Lahmar ${ }^{1}$, F. Lakhdari ${ }^{7}$, O. Larsson ${ }^{57,83}$, J. Lee ${ }^{60}$, J. Licandro ${ }^{82}$, H. Lim ${ }^{60}$, L. López Campano ${ }^{78}$, M.C. Maccarone ${ }^{25,31}$, S. Mackovjak ${ }^{85}$, M. Mahdi ${ }^{1}$, M. Manfrin ${ }^{28,29}$, D. Maravilla ${ }^{62}$, L. Marcelli ${ }^{27}$, J.L. Marcos ${ }^{78}$, A. Marini ${ }^{22}$, K. Martens ${ }^{55}$, Y. Martín ${ }^{82}$, O. Martinez ${ }^{64}$, G. Masciantonio ${ }^{26}$, K. Mase ${ }^{34}$, R. Matev ${ }^{9}$, J.N. Matthews ${ }^{95}$, N. Mebarki ${ }^{4}$, G. Medina-Tanco ${ }^{62}$, L. Mehrad $^{8}$, M.A. Mendoza ${ }^{65}$, A. Merino ${ }^{78}$, T. Mernik ${ }^{17}$, J. Meseguer ${ }^{81}$, S. Messaoud ${ }^{1}$, O. Micu ${ }^{72}$, M. Mignone ${ }^{28,29}$, J. Mimouni ${ }^{4}$, H. Miyamoto $^{28,29, *}$, Y. Miyazaki ${ }^{36}$, 
Y. Mizumoto ${ }^{45}, \mathrm{G}$. Modestino ${ }^{22}$, A. Monaco ${ }^{18,19}$, D. Monnier-Ragaigne ${ }^{10}$,

J.A. Morales de los Ríos ${ }^{80}$, C. Moretto ${ }^{10}$, V.S. Morozenko ${ }^{75}$, B. Mot $^{13}$, T. Murakami ${ }^{39}$,

B. Nadji ${ }^{1}$, M. Nagano ${ }^{36}$, M. Nagata ${ }^{41}$, S. Nagataki ${ }^{57}$, T. Nakamura ${ }^{43}$, T. Napolitano ${ }^{22}$,

D. Naumov ${ }^{73}$, R. Nava ${ }^{62}$, A. Neronov ${ }^{85}$, K. Nomoto ${ }^{55}$, T. Nonaka ${ }^{40}$, T. Ogawa ${ }^{57}$, S. Ogio ${ }^{48}$,

H. Ohmori ${ }^{57}$, A.V. Olinto ${ }^{88}$, P. Orleański ${ }^{71}$, G. Osteria ${ }^{23}$, W. Painter ${ }^{15}$, M.I. Panasyuk ${ }^{75}$,

B. Panico $^{23}$, E. Parizot ${ }^{12}$, I.H. Park ${ }^{60}$, H.W. Park ${ }^{60}$, B. Pastircak ${ }^{76}$, T. Patzak ${ }^{12}$, T. Paul ${ }^{92}$,

C. Pennypacker ${ }^{87}$, I. Pérez-Grande ${ }^{81}$, F. Perfetto ${ }^{23,24}$, T. Peter ${ }^{86}$, P. Picozza ${ }^{26,27,57}$, T. Pierog ${ }^{15}$,

S. Pindado ${ }^{81}$, L.W. Piotrowski ${ }^{89}$, S. Piraino ${ }^{17,25}$, L. Placidi ${ }^{32}$, Z. Plebaniak ${ }^{70}$, S. Pliego ${ }^{62}$,

A. Pollini ${ }^{84}$, E.M. Popescu ${ }^{72}$, P. Prat ${ }^{12}$, G. Prévôt ${ }^{12}$, H. Prieto ${ }^{80}$, M. Putis ${ }^{76}$, J. Rabanal ${ }^{10}$,

A.A. Radu $^{72}$, M. Rahmani ${ }^{7}$, P. Reardon ${ }^{90}$, M. Reyes ${ }^{82}$, M. Rezazadeh ${ }^{88}$, M. Ricci $^{22}$,

M.D. Rodríguez Frías ${ }^{80}$, F. Ronga ${ }^{22}$, M. Roth ${ }^{15}$, H. Rothkaehl $^{71}$, G. Roudil ${ }^{13}$, I. Rusinov ${ }^{9}$,

M. Rybczyński ${ }^{68}$, M.D. Sabau ${ }^{79}$, G. Sáez Cano ${ }^{80}$, H. Sagawa ${ }^{40}$, Z. Sahnoune ${ }^{2}$, A. Saito ${ }^{43}$,

N. Sakaki ${ }^{48}$, M. Sakata ${ }^{42}$, H. Salazar ${ }^{64}$, J.C. Sanchez ${ }^{62}$, J.L. Sánchez ${ }^{78}$, A. Santangelo ${ }^{17}$,

L. Santiago Crúz ${ }^{62}$, A. Sanz-Andrés ${ }^{81}$, M. Sanz Palomino ${ }^{79}$, O. Saprykin ${ }^{74}$, F. Sarazin ${ }^{89}$,

H. Sato ${ }^{42}$, M. Sato ${ }^{52}$, T. Schanz ${ }^{17}$, H. Schieler ${ }^{15}$, V. Scotti ${ }^{23,24}$, A. Segreto ${ }^{25,31}$, S. Selmane ${ }^{12}$,

D. Semikoz ${ }^{12}$, M. Serra ${ }^{82}$, S. Sharakin ${ }^{75}$, T. Shibata ${ }^{50}$, H.M. Shimizu ${ }^{46}$, K. Shinozaki ${ }^{17}$,

T. Shirahama ${ }^{51}$, G. Siemieniec-Oziębło ${ }^{69}$, J. Sledd ${ }^{93}$, K. Słomińska ${ }^{71}$, A. Sobey ${ }^{93}$, I. Stan ${ }^{72}$,

T. Sugiyama ${ }^{46}$, D. Supanitsky ${ }^{62}$, M. Suzuki ${ }^{49}$, B. Szabelska ${ }^{70}$, J. Szabelski ${ }^{70}$, H. Tahi $^{1}$,

F. Tajima ${ }^{38}$, N. Tajima ${ }^{57}$, T. Tajima ${ }^{57}$, Y. Takahashi ${ }^{52}$, H. Takami ${ }^{56}$, M. Takeda $^{40}$,

Y. Takizawa ${ }^{57}$, M.C. Talai ${ }^{3}$, C. Tenzer ${ }^{17}$, O. Tibolla ${ }^{67}$, L. Tkachev $^{73}$, H. Tokuno $^{53}$,

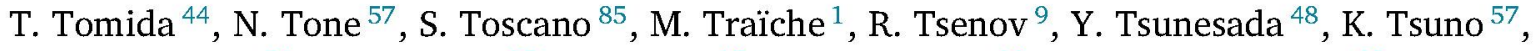

T. Tymieniecka ${ }^{70}$, Y. Uchihori ${ }^{35}$, M. Unger ${ }^{15}$, O. Vaduvescu ${ }^{82}$, J.F. Valdés-Galicia ${ }^{62}$,

P. Vallania ${ }^{28,30}$, G. Vankova ${ }^{9}$, C. Vigorito ${ }^{28,29}$, L. Villaseñor ${ }^{63}$, B. Vlcek ${ }^{80}$, P. von Ballmoos ${ }^{13}$,

M. Vrabel ${ }^{77}$, S. Wada ${ }^{57}$, J. Watanabe ${ }^{45}$, S. Watanabe ${ }^{52}$, J. Watts Jr. ${ }^{90}$, M. Weber ${ }^{15}$,

R. Weigand Muñoz ${ }^{78}$, A. Weindl ${ }^{15}$, T.J. Weiler ${ }^{94}$, T. Wibig ${ }^{70}$, L. Wiencke ${ }^{89}$, M. Wille ${ }^{14}$,

J. Wilms ${ }^{14}$, Z. Włodarczyk ${ }^{68}$, T. Yamamoto ${ }^{42}$, Y. Yamamoto ${ }^{42}$, J. Yang ${ }^{59}$, H. Yano $^{49}$,

I.V. Yashin ${ }^{75}$, D. Yonetoku ${ }^{39}$, S. Yoshida ${ }^{34}$, R. Young ${ }^{93}$, I.S. Zgura ${ }^{72}$, M.Yu. Zotov ${ }^{75}$,

A. Zuccaro Marchi ${ }^{57}$

\footnotetext{
${ }^{1}$ Centre for Development of Advanced Technologies (CDTA), Algiers, Algeria

${ }^{2}$ Dep. Astronomy, Centre Res. Astronomy, Astrophysics and Geophysics (CRAAG), Algiers, Algeria

${ }^{3}$ LPR at Department of Physics, Faculty of Sciences, University Badji Mokhtar, Annaba, Algeria

${ }^{4}$ Lab. of Math. and Sub-Atomic Phys. (LPMPS), Univ. Constantine I, Constantine, Algeria

${ }^{5}$ Laboratory of Theoretical Physics LPT, University of Jijel, Jijel, Algeria

${ }^{6}$ Department of Physics, Faculty of Sciences, University of M'sila, M'sila, Algeria

${ }^{7}$ Research Unit on Optics and Photonics, UROP-CDTA, Sétif, Algeria

${ }^{8}$ Telecom Lab., Faculty of Technology, University Abou Bekr Belkaid, Tlemcen, Algeria

${ }^{9}$ St. Kliment Ohridski University of Sofia, Bulgaria

${ }^{10}$ LAL, Univ Paris-Sud, CNRS/IN2P3, Orsay, France

11 Omega, Ecole Polytechnique, CNRS/IN2P3, Palaiseau, France

${ }^{2}$ APC, Univ Paris Diderot, CNRS/IN2P3, CEA/Irfi, Obs de Paris, Sorbonne Paris Cité, France

${ }^{13}$ IRAP, Université de Toulouse, CNRS, Toulouse, France

${ }^{14}$ ECAP, University of Erlangen-Nuremberg, Germany

${ }^{15}$ Karlsruhe Institute of Technology (KIT), Germany

${ }^{16}$ Experimental Physics Institute, Kepler Center, University of Tübingen, Germany

${ }^{17}$ Institute for Astronomy and Astrophysics, Kepler Center, University of Tübingen, Germany

${ }^{18}$ Istituto Nazionale di Fisica Nucleare - Sezione di Bari, Italy

${ }^{19}$ Università degli Studi di Bari Aldo Moro and INFN - Sezione di Bari, Italy

${ }^{20}$ Dipartimento di Fisica e Astronomia - Universitá Catania, Italy

${ }^{21}$ Consiglio Nazionale delle Ricerche (CNR) - Istituto di Fisica Applicata Nello Carrara, Firenze, Italy

22 Istituto Nazionale di Fisica Nucleare - Laboratori Nazionali di Frascati, Italy

${ }^{23}$ Istituto Nazionale di Fisica Nucleare - Sezione di Napoli, Italy

${ }^{24}$ Università di Napoli Federico II - Dipartimento di Scienze Fisiche, Italy

${ }^{25}$ INAF - Istituto di Astrofisica Spaziale e Fisica Cosmica di Palermo, Italy

${ }^{26}$ Istituto Nazionale di Fisica Nucleare - Sezione di Roma Tor Vergata, Italy

27 Università di Roma Tor Vergata - Dipartimento di Fisica, Roma, Italy

28 Istituto Nazionale di Fisica Nucleare - Sezione di Torino, Italy

${ }^{29}$ Dipartimento di Fisica, Università di Torino, Italy

${ }^{30}$ Osservatorio Astrofisico di Torino, Istituto Nazionale di Astrofisica, Italy

${ }^{31}$ Istituto Nazionale di Fisica Nucleare - Sezione di Catania, Italy

${ }^{32}$ UTIU, Dipartimento di Ingegneria, Rome, Italy

${ }^{33}$ DIETI, Università degli Studi di Napoli Federico II, Nopoli, Italy

${ }^{34}$ Chiba University, Chiba, Japan

${ }^{35}$ National Institute of Radiological Sciences, Chiba, Japan

${ }^{36}$ Fukui University of Technology, Fukui, Japan

${ }^{37}$ Kinki University, Higashi-Osaka, Japan

${ }^{38}$ Hiroshima University, Hiroshima, Japan
} 
${ }^{39}$ Kanazawa University, Kanazawa, Japan

${ }^{40}$ Institute for Cosmic Ray Research, University of Tokyo, Kashiwa, Japan

${ }^{41}$ Kobe University, Kobe, Japan

${ }^{42}$ Konan University, Kobe, Japan

${ }^{43}$ Kyoto University, Kyoto, Japan

44 Shinshu University, Nagano, Japan

45 National Astronomical Observatory, Mitaka, Japan

46 Nagoya University, Nagoya, Japan

47 Institute for Space-Earth Environmental Research, Nagoya University, Nagoya, Japan

48 Graduate School of Science, Osaka City University, Japan

49 Institute of Space and Astronautical Science/JAXA, Sagamihara, Japan

${ }^{50}$ Aoyama Gakuin University, Sagamihara, Japan

51 Saitama University, Saitama, Japan

52 Hokkaido University, Sapporo, Japan

53 Interactive Research Center of Science, Tokyo Institute of Technology, Tokyo, Japan

54 Nihon University Chiyoda, Tokyo, Japan

55 Kavli-IPMU (WPI), The University of Tokyo, Kashiwa, Japan

56 High Energy Accelerator Research Organization (KEK), Tsukuba, Japan

57 RIKEN, Wako, Japan

58 Korea Astronomy and Space Science Institute (KASI), Daejeon, Republic of Korea

59 Ewha Womans University, Seoul, Republic of Korea

60 Sungkyunkwan University, Seoul, Republic of Korea

61 Center for Galaxy Evolution Research, Yonsei University, Seoul, Republic of Korea

62 Universidad Nacional Autónoma de México (UNAM), Mexico

63 Universidad Michoacana de San Nicolas de Hidalgo (UMSNH), Morelia, Mexico

64 Benemérita Universidad Autónoma de Puebla (BUAP), Mexico

65 Centro de Desarrollo Aeroespacial - Instituto Politécnico National (CDA-IPN), Mexico

${ }^{66}$ Universidad Autonoma de Chiapas (UNACH), Chiapas, Mexico

67 Centro Mesoamericano de Física Teórica (MCTP), Mexico

68 Jan Kochanowski University, Institute of Physics, Kielce, Poland

69 Jagiellonian University, Astronomical Observatory, Krakow, Poland

70 National Centre for Nuclear Research, Lodz, Poland

71 Space Research Centre of the Polish Academy of Sciences (CBK), Warsaw, Poland

72 Institute of Space Science ISS, Magurele, Romania

73 Joint Institute for Nuclear Research, Dubna, Russia

74 Central Research Institute of Machine Building, TsNIMash, Korolev, Russia

75 Skobeltsyn Institute of Nuclear Physics, Lomonosov Moscow State University, Russia

76 Institute of Experimental Physics, Kosice, Slovakia

${ }^{77}$ Technical University Kosice (TUKE), Kosice, Slovakia

78 Universidad de León (ULE), León, Spain

79 Instituto Nacional de Técnica Aeroespacial (INTA), Madrid, Spain

80 Universidad de Alcalá (UAH), Madrid, Spain

81 Universidad Politécnia de Madrid (UPM), Madrid, Spain

82 Instituto de Astrofísica de Canarias (IAC), Tenerife, Spain

83 KTH Royal Institute of Technology, Stockholm, Sweden

84 Swiss Center for Electronics and Microtechnology (CSEM), Neuchâtel, Switzerland

85 ISDC Data Centre for Astrophysics, Versoix, Switzerland

86. Institute for Atmospheric and Climate Science, ETH Zürich, Switzerland

87 Space Science Laboratory, University of California, Berkeley, USA

ss University of Chicago, USA

89 Colorado School of Mines, Golden, USA

90 University of Alabama in Huntsville, Huntsville, USA

91 University of California (UCLA), Los Angeles, USA

92 Lehman College, City University of New York (CUNY), USA

93 NASA - Marshall Space Flight Center, USA

94 Vanderbilt University, Nashville, SA

95 University of Utah, Salt Lake City, USA

JEM-EUSO is a space mission designed to investigate Ultra-High Energy Cosmic Rays and Neutrinos $(E>$ $5 \cdot 10^{19} \mathrm{eV}$ ) from the International Space Station (ISS). Looking down from above its wide angle telescope is able to observe their air showers and collect such data from a very wide area. Highly specific trigger algorithms are needed to drastically reduce the data load in the presence of both atmospheric and human activity related background light, yet retain the rare cosmic ray events recorded in the telescope. We report the performance in offline testing of the first level trigger algorithm on data from JEM-EUSO prototypes and laboratory measurements observing different light sources: data taken during a high altitude balloon flight over Canada, laser pulses observed from the ground traversing the real atmosphere, and model landscapes reproducing realistic aspect ratios and light conditions as would be seen from the ISS itself. The first level trigger logic successfully kept the trigger rate within the permissible bounds when challenged with artificially produced as well as naturally encountered night sky background fluctuations and while retaining events with general airshower characteristics. 


\section{Introduction}

Ultra-High Energy Cosmic Rays (UHECR) are observed as Extensive Air Showers (EAS) in the atmosphere surrounding Earth. They are rare events, and the higher their energy, the rarer they get. The greatest mystery surrounding them is their origin, but also their nature remains contentious. High statistics and high quality data are needed to make progress on both fronts, which means scanning the largest possible volume of atmosphere for EAS. The current ground based experiments run up against natural boundaries limiting their expansion and present difficulties when comparing data obtained in northern and southern latitudes, as the fraction of common sky is limited. Therefore, space based instruments observing the atmosphere from above with full-sky coverage have long been considered the logical next step in the evolution of UHECR experiments [1].

The International Space Station (ISS) with its existing infrastructure and support systems is a natural first step on this way into space, and JEM-EUSO [2] is a scientific mission under development with the aim of identifying the astrophysical origin and nature of UHECRs from the ISS. JEM-EUSO detects UHECR induced EAS by looking down onto the earth atmosphere. It has a telescope with a large $\left( \pm 30^{\circ}\right)$ Field of View (FoV) imaging the atmosphere below the ISS onto an array of UV sensitive Multi-Anode Photomultiplier Tubes (MAPMTs) [3]. The MAPMTs (Hamamatsu Photonics R11265-03-M64) have $8 \times 8$ pixels and for readout purposes $2 \times 2$ MAPMTs are grouped into one Elementary Cell (EC). The First Level Trigger (FLT), which is the subject of this article, works at the level of these ECs. Nine ECs form one Photo-Detector Module (PDM), which is the basic unit for the Second Level Trigger (SLT). The Focal Surface (FS) is organized in 137 PDMs. Together these PDMs cover the FS of the telescope with $\sim 3.2 \cdot 10^{5}$ MAPMT pixels. A detailed description of the electronics and data acquisition for JEMEUSO can be found in [4], while a sketch of the structure of the FS is shown in Fig. 1.

The observational concept of JEM-EUSO [5] is based on recording both the fluorescence light emitted during the evolution of EAS as well as the reflected Čerenkov light if the EAS' Čerenkov cone hits a reflective surface as it reaches the ground. EAS from the interaction of UHECRs or neutrinos in the atmosphere will - for $10^{20} \mathrm{eV} \mathrm{EAS} \mathrm{-} \mathrm{typically} \mathrm{result}$ in a few thousand photons detected by the JEM-EUSO detector within a few hundred microseconds. Owing to the large FoV the expected rate of such ultra high energy EAS are approximately one per day. Depending on both the energy and the zenith angle of the EAS, images may be contained inside a single EC or may cross a few PDMs as they are imaged onto across the FS. EAS develop within the lowest $15 \mathrm{~km}$ of the Earth atmosphere, so that their distance to the ISS, which orbits earth at a height above ground of about $400 \mathrm{~km}$, can be considered unchanging whatever an individual EAS' zenith angle happens to be. With that EAS' angular speed across the FS to first order only depends on the EAS' propagation direction relative to the respective FS pixels' direction of view. As EAS traverse the atmosphere at essentially the speed of light, and from the height of the ISS a single square MAPMT pixel's FoV's diagonal measures roughly $750 \mathrm{~m}$ on the ground, it takes about $2.5 \mu \mathrm{s}$ for horizontal EAS' image to traverse the diagonal of a MAPMT pixel. As bandwidth for data transmission from the ISS back to Earth is limited, $2.5 \mu \mathrm{s}$, the so-called Gate Time Unit (GTU), was adopted as the basic unit for digitization at JEM-EUSO. Given the distance between EAS and ISS, JEM-EUSO must be able to detect single photons. The front-end electronics works in single photon-counting mode, which means that $\mathrm{HV}$ and electronics gain are adjusted such that after digitization one digital increment corresponds to one photoelectron (PE) count released from the MAPMT's photocathode.

In this paper we discuss the FLT algorithm specific to the identification of UHECR and neutrino induced EAS. Fig. 2 shows the temporal evolution in units of GTU of the MAPMT signal for typical simulated proton EAS of energy $2 \cdot 10^{20} \mathrm{eV}$, viewed by the JEM-EUSO telescope under an angle of $60^{\circ}$. EAS simulations are performed using the ESAF [6]

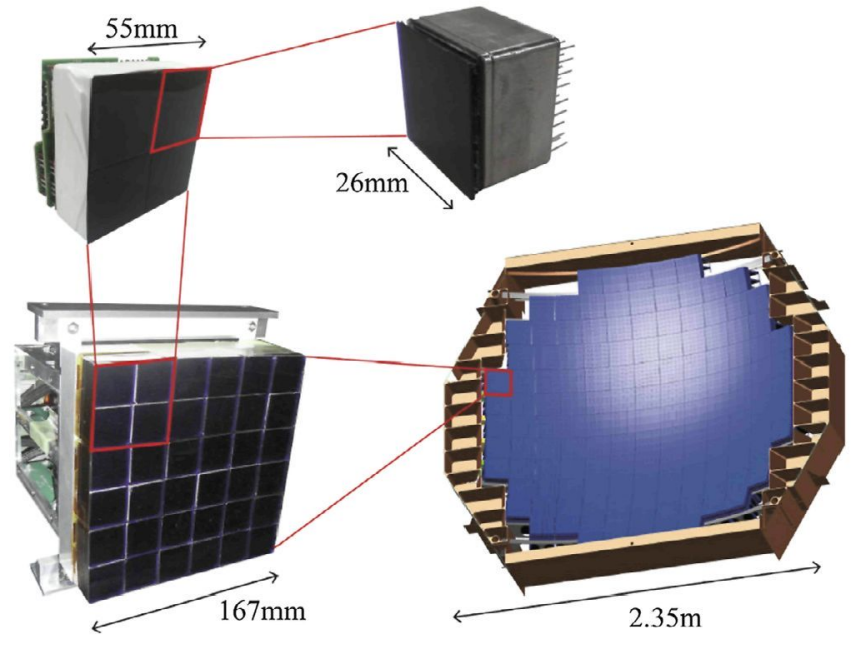

Fig. 1. Structure of the Focal Surface. The $2.5 \mathrm{~m}$ surface is divided in 137 PDM modules. Each PDM is filled with 9 ECs, with 4 MAPMTs each. The bottom left corner shows the prototype of the mechanical structure with 36 MSPMTs installed.

Source: Figure taken from [4]

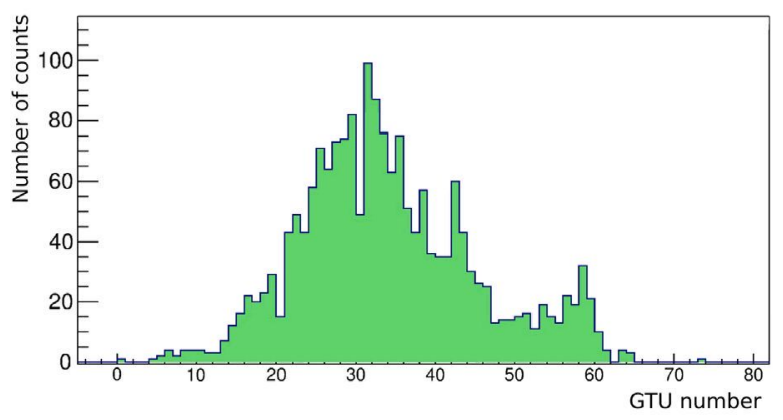

Fig. 2. Light curve (number of photoelectron counts) integrated over the shower profile and plotted over time along the shower axis for a simulated UHECR EAS of $2 \times 10^{20} \mathrm{eV}$ (no background is added). Time is measured in GTUs (see Section 2).

package adapted to the JEM-EUSO instrument. In Fig. 3, the top panel shows the spacial distribution of simulated EAS scintillation light emission projected back onto the Earth's surface for EAS with a common energy of $E=10^{20} \mathrm{eV}$ traversing the atmosphere under zenith angles of (a) $\theta=30^{\circ}$, (b) $\theta=60^{\circ}$ and (c) $\theta=75^{\circ}$. The inset in the lower left corner of this panel puts these showers into the context of the FoV of the whole FS of JEM-EUSO. The bottom panel presents the image of the EAS in (b) as it would be seen by the JEM-EUSO telescope: the optics inverts the direction of motion, and the photon counts per pixel are integrated over the EAS duration which is of the order of $100 \mu \mathrm{s}$.

Looking down from the ISS the FLT has to identify these events in the presence of various backgrounds: UV albedo, transient atmospheric phenomena, and artificial light sources in cities, along transportation networks, and on ships and airplanes. The ISS is moving at about 7.6 $\mathrm{km} / \mathrm{s}$ so that stationary light sources on the ground stay within the FoV of a single pixel for about $70 \mathrm{~ms}$. Such anthropogenic lights, as for example cities, are in the FoV on average during only $\sim 10 \%$ of an ISS orbit [7]. Transient Luminous Events (TLEs) within the atmosphere, like electric discharges (Elves, Sprites, Blue Jets and lightning) as well as meteors will have their own triggering schemes to support a separate science program with JEM-EUSO and will be suppressed by the UHECR and neutrino oriented FLT on the basis of time and light intensity structures. The expected rate of TLEs is $\sim 700 /$ day [8]. The greatest uncertainty is associated with the very slowly varying backgrounds associated with the albedo of the atmosphere: its scattering and reflection of starlight - light reflected from the moon and planets - 

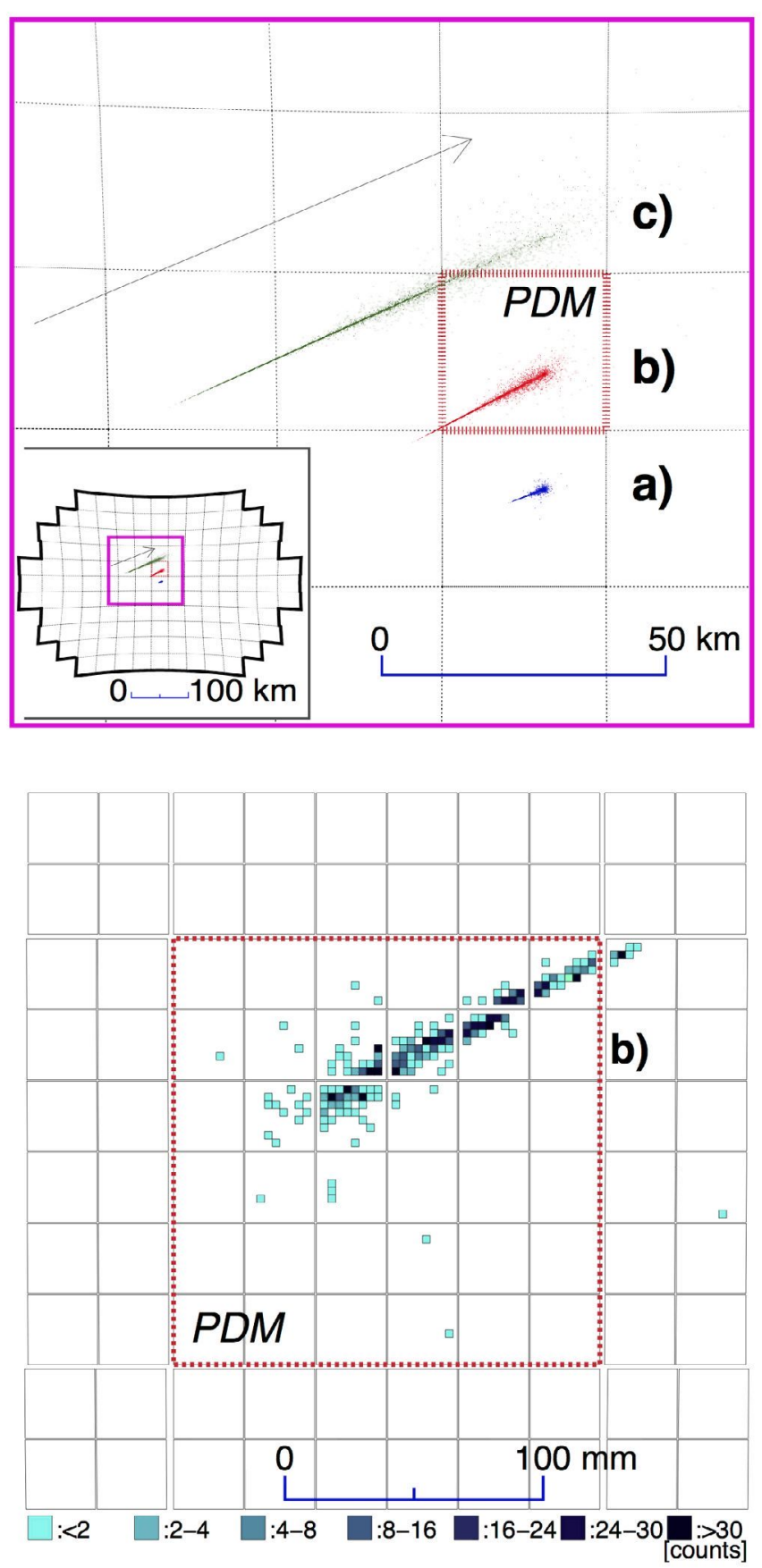

Fig. 3. The top panel shows the 3 EAS with the same energy of $E=10^{20} \mathrm{eV}$ but impinging on the atmosphere under different zenith angles of (a) $\theta=30^{\circ}$, (b) $\theta=60^{\circ}$ and (c) $\theta=75^{\circ}$. The most inclined EAS last $\sim 250 \mu \mathrm{s}$. The arrow indicates the direction of the EAS transit on the FS. The inset on the bottom left and the gray grid shows how the FoV is imaged on the telescope's FS. The distance scale on this panel refers to the distance the shower develops over as projected onto the Earth surface. The bottom panel shows the image (inverted by the optics) on shower (b) as recorded (integrated over time) by the JEM-EUSO telescope. The distance scale here refers to distance on the FS. The regions enclosed by thick dashed lines in both panels refer to the same PDM. Image taken from [7]. UV background is not added in these plots.

and airglow. On a clear night the resulting diffuse flux of such photons is expected to amount to between 300 and 1000 photons $\mathrm{m}^{-2} \mathrm{~ns}^{-1} \mathrm{sr}^{-1}$, an expectation derived from existing measurements $[9,10]$.

In this paper we show the results of the offline testing of the current JEM-EUSO FLT concept using data from three main sources rather than simulation: data collected during a high altitude balloon flight over Canada (EUSO-Balloon), laser shots recorded in coincidence with a ground based JEM-EUSO prototype (EUSO-TA), and data taken in a laboratory setup where realistic background scenarios can be explored (EUSO@TurLab).

\section{Technical requirements}

As mentioned before, working on the ISS imposes severe bandwidth constraints on data transfer to the ground. On top of that there is a $\sim 1 \mathrm{~kW}$ limit on power consumption for the whole telescope, including the readout and trigger electronics, high voltage for the MAPMTs, and monitoring. This constraint for example means that triggering cannot be substituted for by massive only computing. Data rate considerations also played into choosing a GTU of $2.5 \mu$ s for digitization in time and the number of 128 GTUs per packet, meaning that each event record will contain the timing evolution of a signal over a time span of $320 \mu \mathrm{s}$.

Since the ISS is far above the parts of the atmosphere where EAS develop, the MAPMTs have to be able to detect faint signals using photon-counting. Therefore, 8 bit full scale per pixel is sufficiently large. Under these conditions the total data rate from the telescope before the FLT would be of order $3.2 \cdot 10^{5} \mathrm{pixel} / \mathrm{FS} \times 4 \cdot 10^{5} \mathrm{GTU} / \mathrm{s} \times 8 \mathrm{bit} / \mathrm{pixel} \approx 1$ Tbps. To achieve the required overall data reduction of $\sim 3 \cdot 10^{6}$ the FLT will have to reduce the trigger rate to $\sim 1 \mathrm{~Hz} / \mathrm{EC}$, and the PDM based second level trigger to $\sim 0.1 \mathrm{~Hz} / \mathrm{FS}[11,12]$.

In the following section, specifics of the FLT logic aimed at detecting UHECR and neutrino induced EAS, which are the main scientific objective of the JEM-EUSO mission, are considered. While TLEs and other bright but slow atmospheric events are part of the exploratory objectives of the mission, we will not consider them here.

\section{The 1st trigger level: persistency tracking trigger}

Persistency is a measure of how long in time a signal "persists" or stays within the FoV of a particular MAPMT pixel. As detailed above a GTU roughly reflects the time horizontal EAS right under the ISS need to travel the diagonal of a MAPMT pixel. To cross the FoV of an EC , where the FLT operates, takes up to $45 \mu \mathrm{s}$ for EAS, milliseconds for lightning, hundreds of milliseconds for meteors, and seconds for cities or airplanes. These differences in persistency and the fact that the signal moves from pixel to pixel as EAS pass through the FoV of the telescope were exploited in designing the FLT logic, which is described in [13]. Here we give a summary of how an EAS trigger is formed at the EC/FLT level.

Unless EAS develop along the line of sight of the telescope, the image of their fluorescence trails in the atmosphere can be tracked across the FS. Tracking discriminates EAS images against accidental coincidences of background light fluctuations.

To decide if a single MAPMT pixel is seeing signal above a slowly varying, non-negligible background, this background level has to be estimated. Two different approaches were pursued: The pixel-based estimate sets one threshold for a whole MAPMT. To obtain this threshold the average over a 128 GTU data packet is calculated for each pixel in the MAPMT, and the maximum of these 64 averages becomes the background estimate and threshold for triggering in the next 128 GTU packet. Stationary or slowly moving anthropogenic light sources within the atmosphere are automatically suppressed by this method. The groupbased estimate divides the whole EC into 32 groups of $2 \times 4$ pixels, calculates the $128 \mathrm{GTU}$ averages per group, and chooses the maximum of those 32 averages as the threshold for all pixels in the EC during the next 128 GTU packet. The threshold here is a digital value as all calculations are done after digitization. No analog thresholds are used. The results presented in this paper are based on the pixel-based estimate which turned out to be better performing overall.

For tracking purposes each MAPMT's pixels are grouped into square $3 \times 3$ cells. Each pixel belongs to more than one cell, but since cells do not span MAPMT borders, pixels along the edges and in particular at the corners of each MAPMT belong to fewer cells than the central 
pixels. The first panel in Fig. 4 shows three such overlapping cells on a single MAPMT's pixel grid outlined in dark orange. Since it includes neighboring MAPMTs' pixels, the $3 \times 3$ pixel patch surrounded by the dashed gray line on the other hand does not constitute a cell. A total of 36 cells exists within each MAPMT. Each pixel for which its digitized signal in a certain GTU of a 128 GTU packet surpasses the threshold value $n_{\mathrm{thr}}^{\text {pix }}$ for that MAPMT (determined from the data in the preceding $128 \mathrm{GTU}$ ) contributes to each of the cells it participates in. Apart from the pixel level signal threshold there also is a cell level signal threshold $n_{\text {thr }}^{\text {cell }}$. For a typical background level of one PE per GTU per pixel these thresholds $n_{\mathrm{thr}}^{\text {pix }}$ and $n_{\mathrm{thr}}^{\text {cell }}$ would normally be set to 3 and 31, respectively.

Persistency at the pixel level is evaluated based on two more parameters that unlike those introduced above do not depend on the background situation: a pre-determined range of consecutive GTUs $N_{\text {pst }}$ over which persistency is to be evaluated, and a limit $N_{\text {ctd }}$ on the number of GTUs within that range for which pixels in the cell are above threshold. Standard values for the pixel related parameters $N_{\mathrm{pst}}$ and $N_{\text {ctd }}$ are 5 and 3 GTUs, respectively. These values were determined by means of simulations of EAS signals and of preliminary tests on MAPMT fluctuations with the aim of keeping EAS signals and rejecting background fluctuations. Persistency of a signal at the EC level is also monitored and similarly checked by two parameters for a maximum allowed number of GTUs above threshold $N_{G T U}^{t h r}$ in a GTU range $N_{G T U}$. Too many GTUs with signal indicate high persistency, which is the hallmark of non-EAS induced events like lightning or meteors. This GTU range is started at the GTU in which for the first time a cell threshold is surpassed. Typical values for EAS identification are $N_{G T U}=73$ and $N_{G T U}^{t h r}=72$. These two values were decided according to the following considerations. $N_{G T U}$ is determined by the number of GTUs remaining after the first trigger till the end of the packet. For technical reasons it was decided to have the trigger at GTU 55 of a packet. The $N_{G T U}^{t h r}$ indicates that all the events are accepted unless the PDM continues triggering every GTU after the first trigger till the end of the packet. Both values will be fine tuned in future, if needed. In particular $N_{G T U}^{\text {thr }}$ can be easily shortened by a few GTUs without impacting the trigger efficiency on EAS.

As mentioned above power consumption is a major constraint on the ISS. The current implementation of the FLT was programmed into and tested on a Xilinx Virtex6 model XC6VLX240T [14] FPGA. Given that it required only $\sim 7 \%$ of this FPGA's resources to accommodate the logic needed for $1 \mathrm{EC}$, it is expected that one such FPGA can host all $9 \mathrm{ECs}$ belonging to one PDM. The graphic in the following figure reflects the FPGA architecture in its reference to the FPGA's various adders.

Fig. 4 uses an event recorded by EUSO@TurLab ${ }^{1}$ to illustrate how a FLT is formed. The background estimate was derived from the preceding GTU packet as $n_{\mathrm{thr}}^{\mathrm{pix}}=5$ and $n_{\mathrm{thr}}^{\text {cell }}=65$; the average background light level for that packet was of order 3.4 PE/GTU/pixel. Using the standard values of $N_{\mathrm{ctd}}=3$ and $N_{\mathrm{pst}}=5$, the graphic follows the cell's pixels' recorded PE counts for an EAS-like event created by a line of LEDs mimicking an almost vertical shower mostly staying in the FoV of the cell's central pixel. In the first panel of Fig. 4 the cell is highlighted in dark orange on the MAPMT's pixel map. The next five sub-panels of the figure after the MAPMT pixel overview show two pixel maps each for that same cell. The five sub-panels represent the five successive GTUs following the cell's first threshold crossing. The pixel map on the left in each sub-panel shows the raw PE counts recorded per MAPMT pixel in the respective GTU. Using the estimated $n_{\mathrm{thr}}^{\mathrm{pix}}=5 \mathrm{PE}$ background as threshold then leads to the pattern of threshold-crossing pixels displayed on the right of the sub-panel with each pixel's background subtracted PE signal estimates. The sum of that signal above background is then compared to the cell threshold $n_{\text {thr }}^{\text {cell }}=65 \mathrm{PE}$. In summary: for the chosen cell and its five GTUs after the cell's threshold crossing, at least one pixel in the cell crosses the pixel threshold for each GTU, and the total signal strength accumulated within this cell in each GTU is enough to
Table 1

Variability of the signal expected for JEM-EUSO due to different light sources in the FoV of the telescope, ranging from steady UV nightglow to localized and impulsive light bursts such as cities, EAS, meteors. The maximum luminosity of meteors is here defined by the saturation of the front-end electronics.

\begin{tabular}{lllll}
\hline Light source & $\begin{array}{l}\text { Intensity } \\
\text { (cts/pix/GTU) }\end{array}$ & Duration & Extension & Variability \\
\hline UV glow & $0.5-5$ & Orbit & EC & Water, soil, cloud \\
Urban & $3-30$ & Seconds & pixel - EC & Village - city \\
EAS & $3-30$ & $\sim 100 \mu s$ & PMT (track) & EAS energy \\
Meteor & $3-100$ & Seconds & EC (track) & Magnitude \\
\hline
\end{tabular}

contribute to the EC wide evaluation of the event. Therefore, at GTU step 5 the corresponding adder ( $\mathrm{T}$ ) is incremented. The EC-wide check with regard to the GTUs during which the signal passes through all the other EC cells is summarized in the last panel, where the content of the adder $\mathrm{T}$ is finally checked before a FLT is issued (or not) to the PDM for second level trigger purposes.

Persistence is the main concept behind the FLT implementation. In the following section we will discuss how this current implementation performs in the presence of background, using data recorded with EC modules in dedicated experiments reflecting different aspects of space based EAS observation in the Earth atmosphere.

\section{FLT tests using experimental data}

The trigger efficiency as a function of EAS energy (commonly referred to as the trigger efficiency curve) captures an important aspect of the experiment's sensitivity. Several publications already discussed the expected JEM-EUSO sensitivity given the current FLT implementation, but so far they were purely based on MC simulations $[5,7,15]$. These simulations all assumed Poisson fluctuations on a UV background intensity that is constant and uniform across the FS.

In this section we report on tests performed offline using data taken with actual ECs in three very different environments, each addressing specific challenges JEM-EUSO is expected to face during observation from the ISS: data collected by the EUSO-Balloon flight in 2014 [16], measurements performed by EUSO@TurLab at TurLab [17], and observations in coincidence with a Telescope Array (TA) air fluorescence detector by EUSO-TA [18]. These data sets allow to test the trigger system in very different and complementary ways. EUSO@TurLab provides the possibility to control lighting and create realistic event patterns and persistences, EUSO-Balloon takes data under space-like conditions, and EUSO-TA allows comparison with a well calibrated existing groundbased fluorescence detector.

\subsection{Tests with TurLab measurements}

The two main aspects of the FLT that were tested at TurLab, located at the Physics Department of the University of Turin (Italy), were the adequacy of its background estimation and the ability to trigger on EAS while suppressing other signatures, such as cities, meteors, lightnings, discontinuities in the luminosity due to the presence of clouds, variation in soil condition, moon phase, etc. All these phenomena have variable intensity, duration and extension. Table 1 gives typical values expected for JEM-EUSO for a subset of these conditions which have been reproduced at TurLab to test the trigger logic.

Being $15 \mathrm{~m}$ under ground, the ambient light level in the TurLab laboratory [17] is several orders of magnitude lower than that of the darkest night sky. Using artificial light sources therefore puts the ambient light levels as well as the distribution of light in the lab under the control of researchers.

At TurLab a rotating tank of $5 \mathrm{~m}$ diameter provides the stage on which light emitting as well as light reflecting installations are made. EUSO@Turlab consists of one EG hung off-center from the ceiling above this rotating tank. While in principle the EC can be moved radially it was 

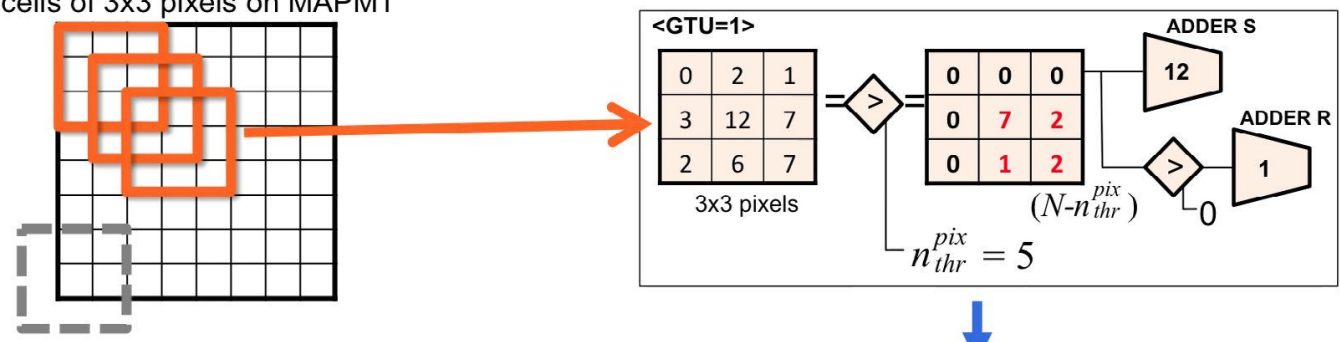

$+\frac{1}{2}$

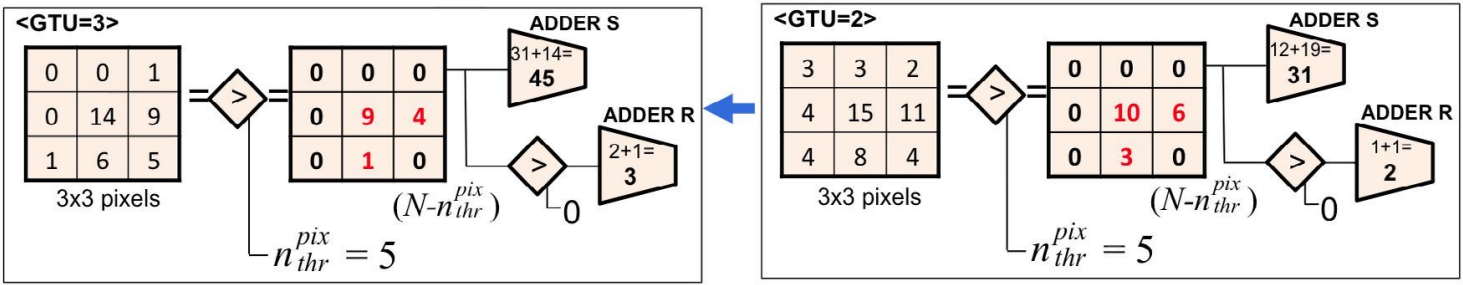

$\downarrow$

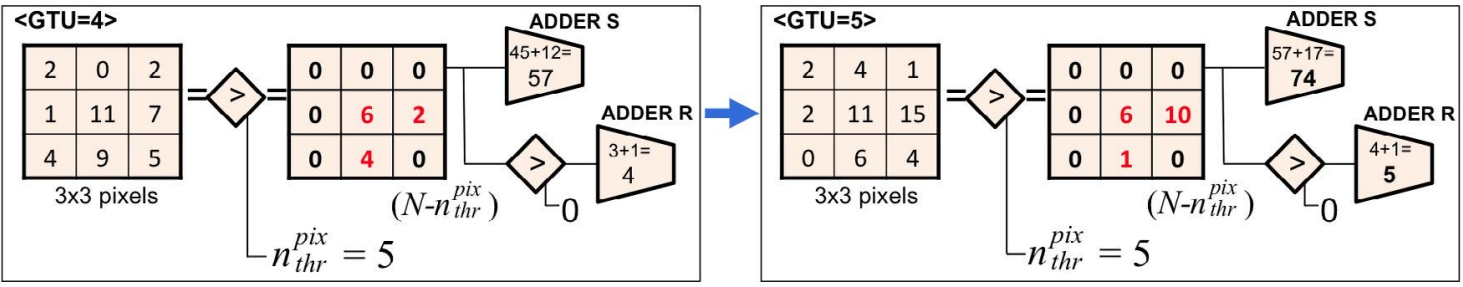

At GTU=5 (=N $\left.N_{p s t}\right)$ :

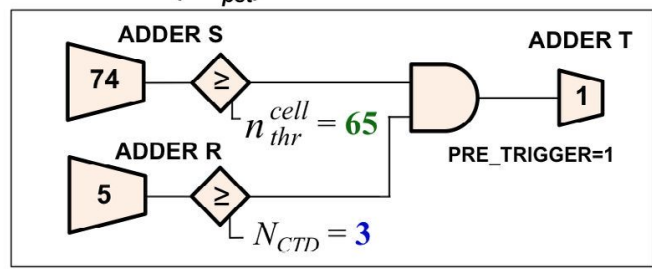

All the sequence repeated for:

$\mathrm{N}_{\mathrm{GTU}}=2 \ldots 6,3 \ldots 7, \ldots, 73 \ldots 77$

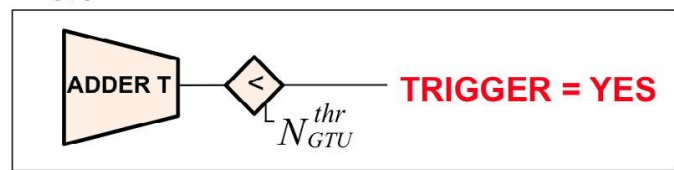

Fig. 4. The FLT implementation at the level of the $3 \times 3$ cells. See text for details.

kept at a radius of roughly $2 \mathrm{~m}$ from the center of the tank. The optics imaged $1 \mathrm{~cm}^{2}$ on the tank's surface onto one pixel $2 \mathrm{~m}$ above the tank surface, giving it a FoV of the order of $10^{-5} \mathrm{sr}$, which is only one order of magnitude larger than a JEM-EUSO's pixel. This means that if the adjustable speed of the tank rotation were to be around two minutes, the time it takes a stationary source on the tank surface to cross a pixel would be the same as it will be for JEM-EUSO looking down on Earth from the ISS

As outlined before, the data acquisition (DAQ) in JEM-EUSO will be a seamless sequence of 128 GTU long packets. At EUSO@TurLab the EC's ASIC is read out by a test board which transfers the data to a PC, and this system both limits a data packet to 100 GTUs and imposes a $\sim 50 \mathrm{~ms}$ deadtime between two consecutive packet acquisitions. In other words, at EUSO@TurLab 100 GTUs = 250 $\mu$ s of data are taken every $~ 50 \mathrm{~ms}$, and a stationary light source on the tank surface would have moved through $50 \%$ of the FoV of a pixel during that deadtime if the tank rotated with a period of $2 \mathrm{~min}$. It was not possible to synchronize the DAQ with the tank rotation, because it was not foreseen by the control system of the tank. Naturally, the synchronization would have allowed to determine exactly the location in the tank responsible for each trigger.

Fig. 5 shows the various components of the TurLab setup. It shows the EC suspended from the ceiling, and various installations on the tank surface designed to emit or reflect light in ways that mimic both anthropogenic and natural lighting situations as they would be seen by JEM-EUSO from the ISS (see Table 1). Light scattered or reflected

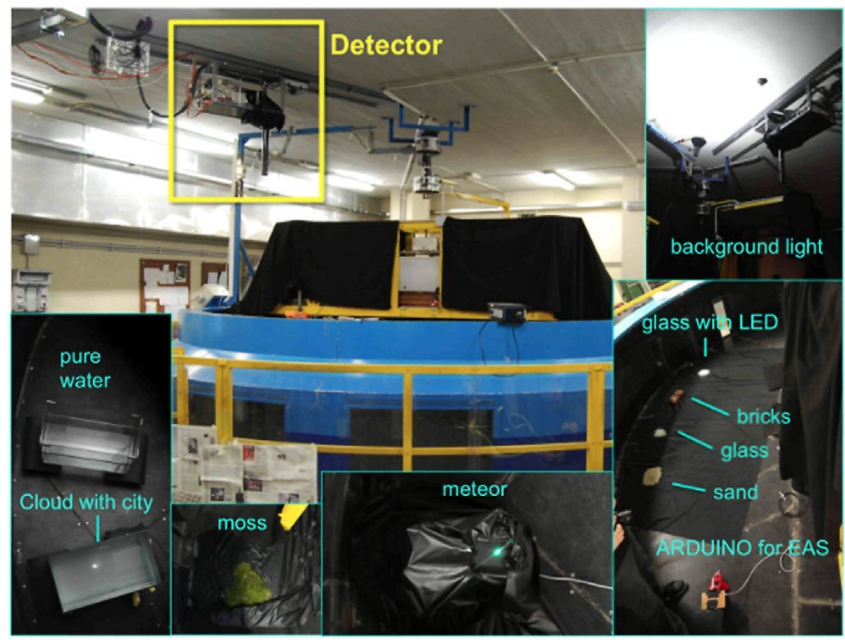

Fig. 5. The TurLab rotating tank. The black tube on the ceiling shows the collimator of the experimental setup used to mimic the JEM-EUSO telescope. Light sources and materials used to mimic other phenomena are shown as well. (For interpretation of the references to color in this figure legend, the reader is referred to the web version of this article.) 
from the atmosphere back into space - the Earth's albedo - depends on atmospheric conditions like e.g. the presence or absence of clouds, the reflectivity of the Earth's local surface, and lighting conditions like the phase of the moon ${ }^{2}$ or the presence and density of human habitation.

In Fig. 5 one can also see how sand, moss, ground glass, pure water, and a brick were used to mimic the reflection of night-sky light from soils, forests, snow, water, and rocky surfaces, respectively. Water clouded by dissolved particles and illuminated from below is used to mimic clouds, and if illuminated from below cloud cover over e.g. a city. An oscilloscope screen displaying Lissajous traces mimics meteor tracks. As lighting can be controlled, the TurLab tank allows to verify the performance of the background estimation under realistically varying lighting conditions. Figs. 6 and 7 show two examples of recordings of such features with EUSO@TurLab.

In Fig. 6 LED light reflected from ground glass is used to simulate the distributed individual light sources of a city. Each of the four frames in each sub-panel refers to one of the four MAPMTs in the EC. The upper panels show the respective MAPMT's integrated PE counts, in other words the light curve of a city passing through the FoV of the EC. The city entering and exiting the MAPMT's FoV as time progresses is clearly visible in each MAPMT's light curve. The lower row shows 2D pixel maps for the EC's MAPMTs, with the PE counts per pixel for just one GTU on the left, 10 GTUs in the center, and 100 GTUs on the right. The red lines in the light curves show the range of GTUs that are used, with the single GTU pixel maps being the first GTU under both red lines; the start times of this integration is the same for all three ranges. Fig. 7 shows the data recorded while passing over the oscilloscope repeating a straight line Lissajous figure taking about one second to complete. While the complete picture emerges after integrating over 1500 GTUs (right panel), the signal still is contained in a single pixel when integrating over only 10 GTUs (center panel).

An Arduino board [19] controlling a line of 10 white LEDs was used to mimic a single EAS propagating through the atmosphere at the speed of light, resulting in a total duration of about 40 GTUs. As can be seen in Fig. 8 this signal does no longer stay within one pixel during 10 GTUs, with the center of light moving visibly between subsequent GTUs.

The FLT estimates the background for the current data packet from the data collected in the preceding data packet. To mitigate possible adverse effects of the DAQ-imposed deadtime between the acquisition of consecutive packets on background estimation the tank rotation was slowed to complete one rotation in $9 \mathrm{~min}$, reducing the offset of a stationary light source between consecutive acquisition packets from $50 \%$ of a pixel to roughly $10 \%$ of a pixel; with the JEM-EUSO DAQ a $128 \mathrm{GTU}$ offset at ISS speed would correspond to $0.5 \%$ of the pixel size projected onto the ground. Given the deadtime between the 100 GTU acquisition packets a total of $\sim 3 \mathrm{~s}$ of data is collected during one $9 \mathrm{~min}$ rotation.

The DAQ at TurLab collects that data "as is": it simply reads out the PE counts for each MAPMT pixel in each GTU from the EC's ASIC and writes them to disk. The subsequent trigger simulation is then implemented in $\mathrm{VHDL}^{3}$ according to the schema described in [13].

The light collected on one of the EC's MAPMTs during a complete 9 min rotation of the tank is shown in the upper panel of Fig. 9. Changes in the background light level are clearly seen, and the various contraptions that precipitate them are labeled in the figure. The two bridges refer to the footbridges to cross the tank which, despite being covered by some black fabric, are a source of quite variable light reflection. In general the black fabric was used to make as dark as possible specific regions of the tank to help increase the dynamic range of the light intensity seen by the MAPMTs during the tank rotation. Pure water in a little transparent tank was used to mimic a mirror-like condition which induces much higher reflection. The yellow bar is a pole on the rotating tank which passes a few $\mathrm{cm}$ below the collimator,

\footnotetext{
${ }^{2}$ Fluorescence observation of EAS is not possible during daytime.

3 Very high speed integrated circuit Hardware Description Language.
}

Table 2

Number of triggered and extracted cosmic-ray-like-track events in various background photon level conditions. $N_{\text {PEave. } / 4 \times 4 \text { pix }}$ indicates the average background level expressed in counts per pixel per GTU evaluated on a $4 \times 4$ pixel-box during the preceding packet of data, where no Arduino event was extracted.

\begin{tabular}{lll}
\hline$N_{\text {PEave./4×4pix }}$ & $N_{\text {triggered }}$ & $N_{\text {extracted }}$ \\
\hline 0.21 & 24 & 24 \\
0.36 & 13 & 13 \\
0.56 & 15 & 15 \\
0.78 & 21 & 21 \\
1.00 & 26 & 26 \\
1.23 & 16 & 16 \\
1.47 & 26 & 26 \\
1.69 & 22 & 22 \\
1.93 & 20 & 20 \\
2.11 & 31 & 31 \\
\hline
\end{tabular}

thus filling a significant portion of the FoV of the detector for a short time. The second panel of Fig. 9 shows the average PE count in the preceding 100 GTU packet for the pixel with the maximal average count in that same MAPMT, which is the value used for the threshold setting in the current packet. The final panel shows how the trigger simulation reacted to this input. It shows when FLTs were issued based on signals in that MAPMT. Almost all triggers coincide with passing over the Arduino driven LED chain as it should be; the one that is not is due to a specific location near one of the two bridges crossing the tank where the variations of light reflection were still too fast to be compensated by the slower rotation of the tank. Fig. 10 shows top and bottom plots of Fig. 9 for all four MAPMTs. A similar response is obtained for all four MAPMTs all along the rotation.

To directly assess the impact of background on the FLT trigger scheme for EAS dedicated measurements were made at TurLab with the tank rotation stopped and the EC stationary above the Arduino driven white LED strip simulating EAS. Ambient light levels then controlled the background to the LED induced signal. These ambient light levels were varied between 0.1 and 2.0 PE per pixel and per GTU, reflecting expectations for typical ISS observation background. The Arduino EAS were generated $1 \mathrm{~ms}$ apart in order to reduce the probability of recording such Arduino EAS in consecutive data packets, in which case the first EAS would set the background level for the second EAS. As the DAQ for EUSO@TurLab was not synchronized with track timing in the Arduino, extracting the packets containing a complete Arduino track required some event selection.

This selection started from a $4 \times 4$ pixel box in that MAPMT which contained the brightest part of the Arduino LED simulated EAS. The stationary tank was oriented such that the Arduino LEDs were all within the field of view of a single MAPMT and the Arduino EAS were always crossing the same MAPMT pixels.

The LED sequence for these Arduino EAS was kept stable with $\sim 30$ $\mathrm{PE}$ at maximum, which corresponds to recording a $\sim 6 \times 10^{19} \mathrm{eV}$ EAS in JEM-EUSO. A mask above the LEDs was used as an aperture to avoid unwanted reflections of LED light from nearby structures above the tank. The voltages supplied to the LEDs were also adjusted to dim the LEDs that were closer to the ends of the strip in an effort to provide a realistic EAS profile when the Arduino board sequentially lights up the LEDs in the strip.

If the PE count in the $4 \times 4$ pixel box smoothed over 5 GTUs exceeded the corresponding background estimate by more than $4 \sigma$, the data were considered an Arduino EAS candidate. Such a candidate would subsequently be rejected if the excess occurred only in the first or last five GTUs of a 100 GTU data packet, or if the preceding data packet also contained an Arduino EAS candidate. Fig. 11 shows PE counts for the relevant MAPMT over time in GTU units. The left panel shows a typical event produced by the LED strip without background. This highlights the event's original shape. The right one is a similar event produced under high background.

Events selected by this procedure were then fed into the VHDL trigger simulation. Table 2 shows that all the selected Arduino EAS 

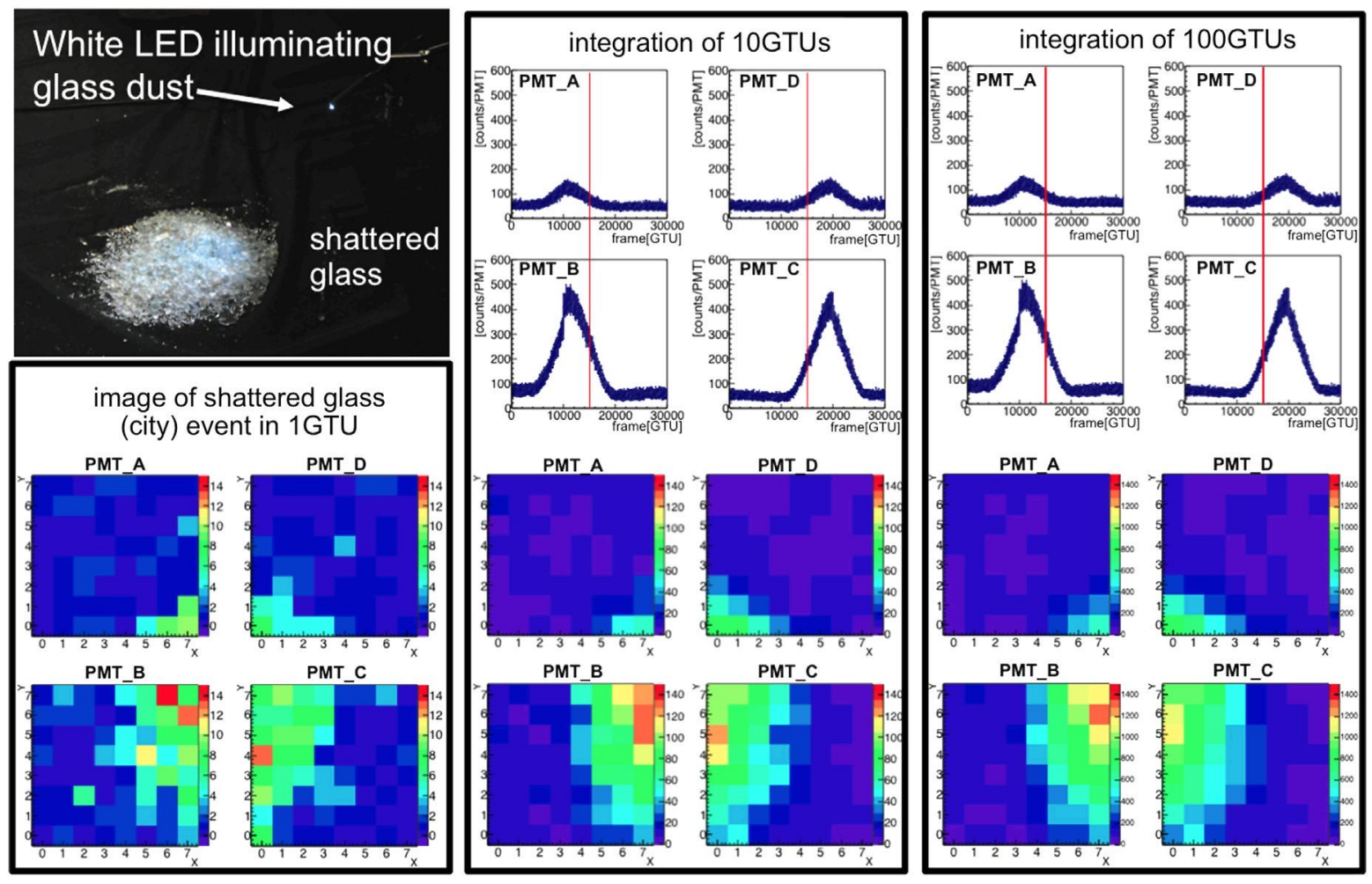

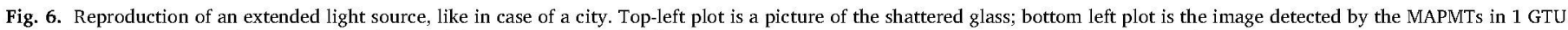

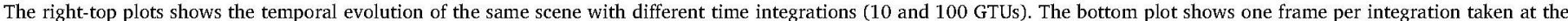
time indicated by the red line in the above plots. (For interpretation of the references to color in this figure legend, the reader is referred to the web version of this article.)

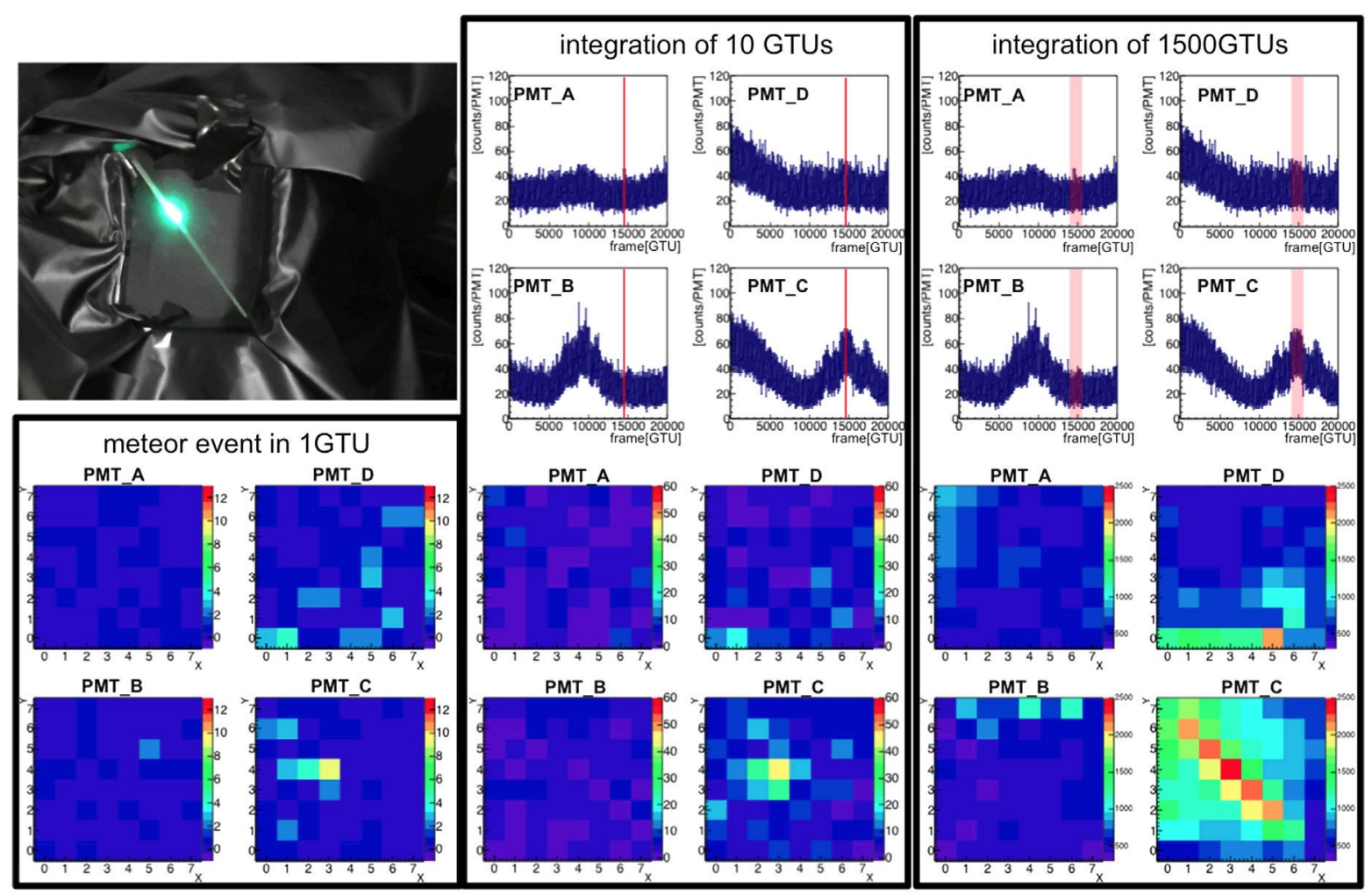

Fig. 7. Reproduction of a meteor-like track. See Fig. 6 for details about the meaning of each plot in the figure.

also triggered in the FLT simulation. This together with the fact that when observing the rotating tank with its various implementations of atmospheric as well as ground-based light sources, albedo effects and generally varying background light levels gave rise to only a few spurious triggers under very specific conditions gives confidence that the current FLT implementation is ready for deployment in JEM-EUSO. 


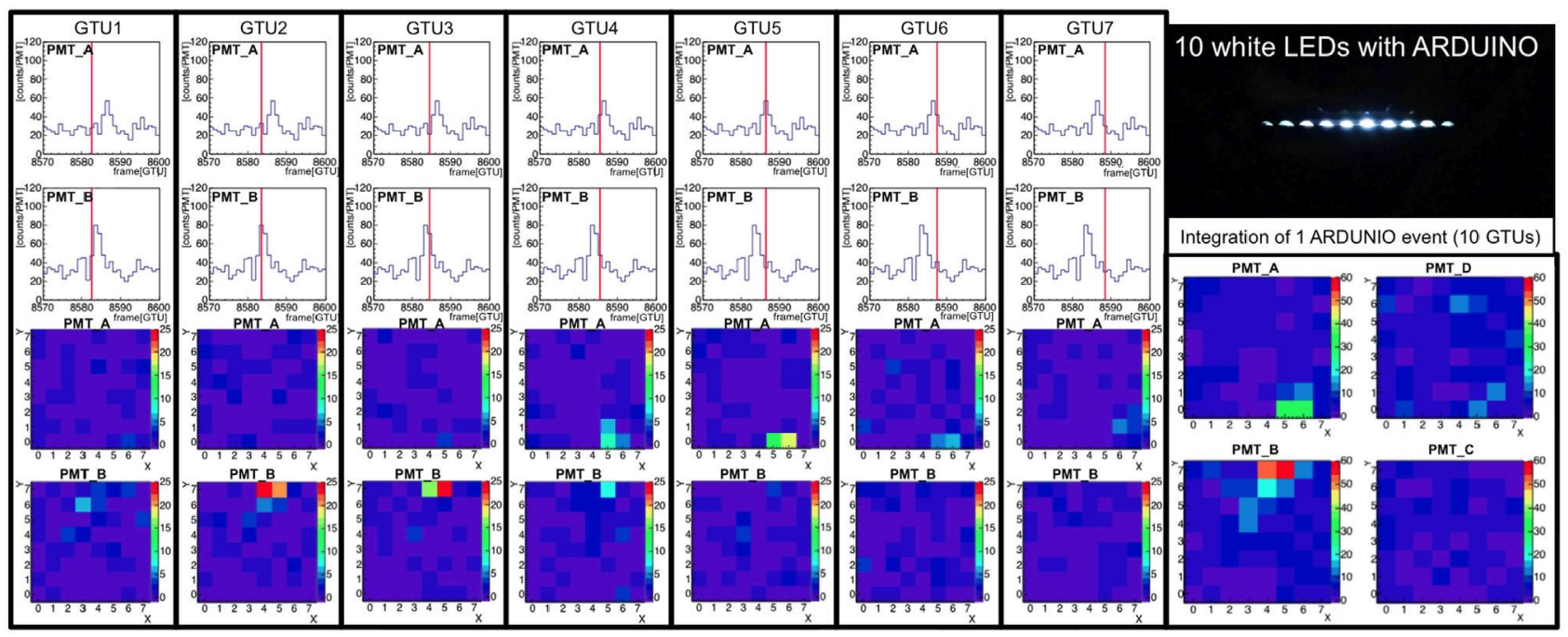

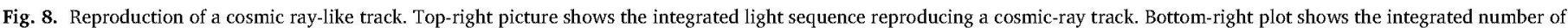

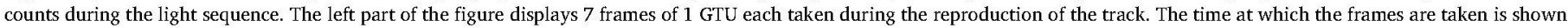
in the above corresponding plots which present the time evolution of the total number of counts recorded by the MAPMT.

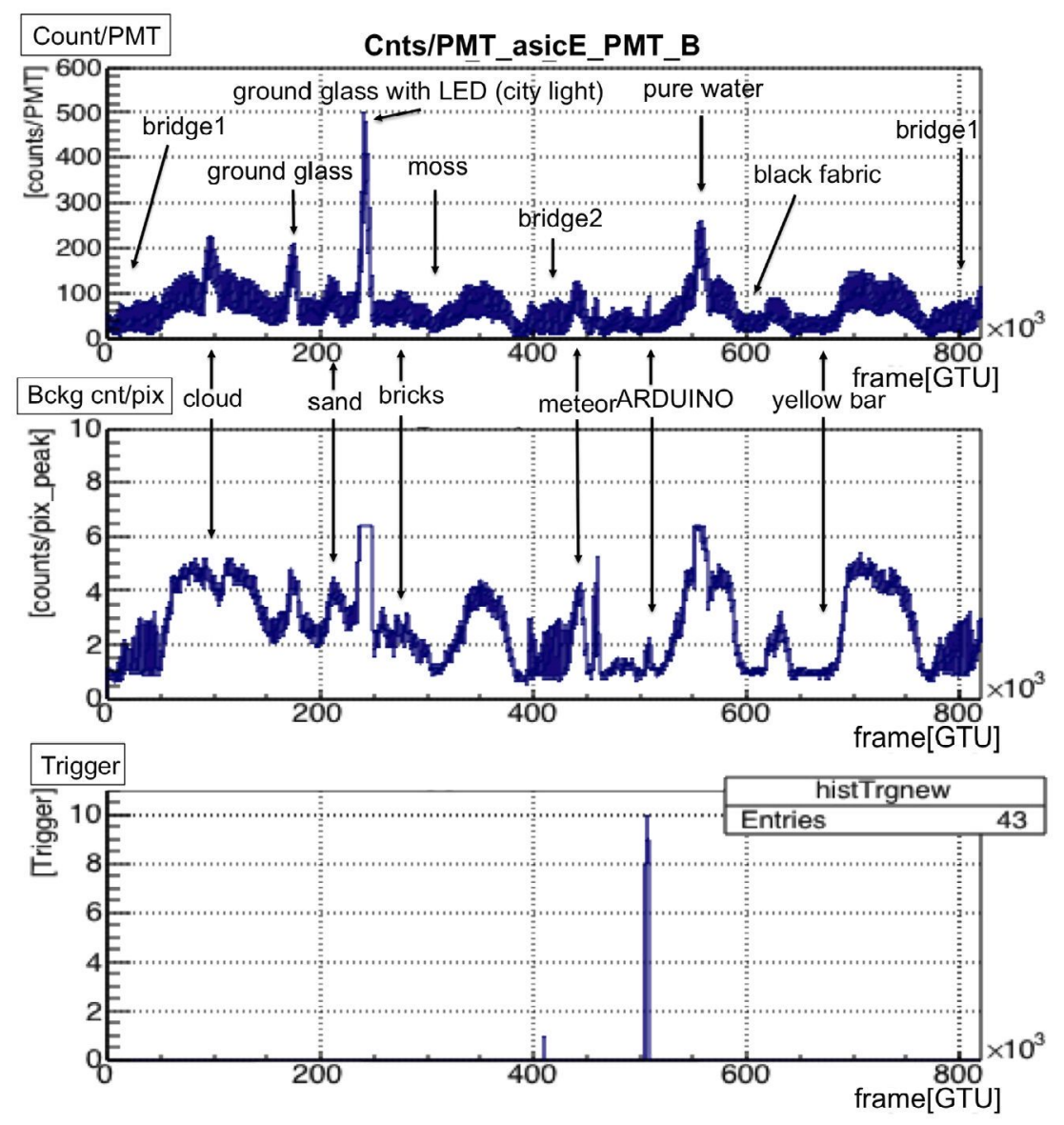

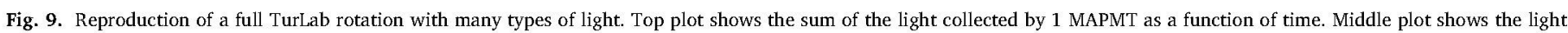

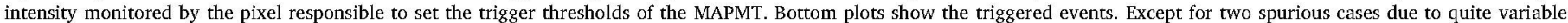

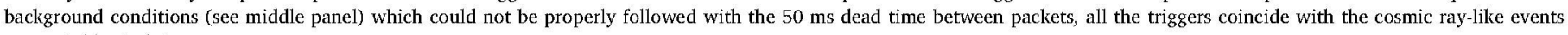
generated by Arduino.

\subsection{Tests with EUSO-Balloon data}

The EUSO-Balloon [20,21] data taken during a $5 \mathrm{~h}$ flight at $38 \mathrm{~km}$ altitude in the vicinity of Timmins in Canada provides another testbed for the FLT. Again the adequacy of the newly adopted background estimation method with respect to keeping the trigger rate within the permissible bounds in the presence of artificially and naturally encountered fluctuations in the background lighting conditions as well as the FLT's ability to trigger on relevant optical phenomena was studied. While at TurLab the optics and speed could be adjusted to 

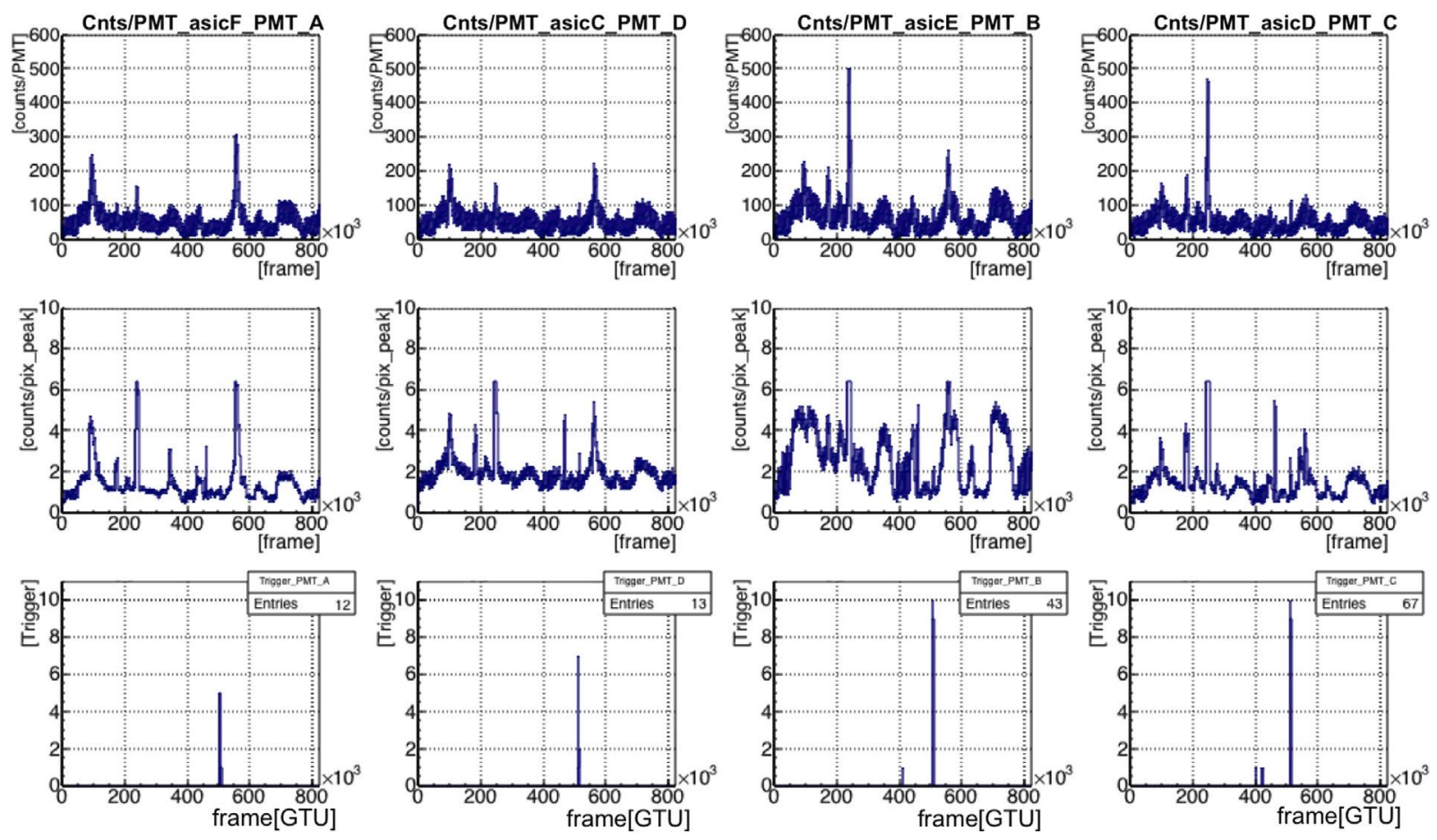

Fig. 10. Reproduction of a full TurLab rotation with many types of light sources as shown in Fig. 9 for the entire EC.
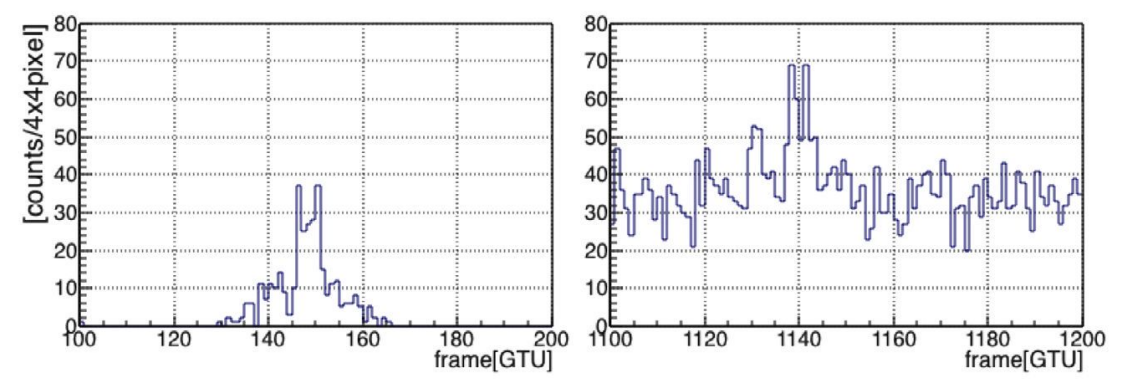

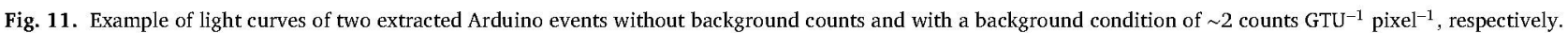

match event duration and persistence in a pixel's FoV for the various phenomena recreated there, the EUSO-Balloon's speed and trajectory could not be controlled to that extent. On the other hand EUSO-Balloon looked down on a real Earth environment just as JEM-EUSO will, albeit from a much closer distance than the ISS. Thus were TurLab strove to be realistic in an artificial environment, EUSO-Balloon was looking at realistic settings compromising on perspective. Thus they each capture different aspects of the challenges presented to an FLT operating at JEMEUSO.

In flight the EUSO-Balloon optics imaged a $60 \mathrm{~km}^{2}$ surface area onto one full PDM with its $9 \mathrm{ECs}$ and a total of 36 MAPMTs. Just as at TurLab the data acquisition did not allow to record data continuously, but took $128 \mathrm{GTU}$ data packets at $18 \mathrm{~Hz}$, translating into $320 \mu \mathrm{s}$ of data recorded every $\sim 55 \mathrm{~ms}$. Data taking on the balloon was organized into runs. A new run would be started after either 200 or 2000 data packets had been recorded. In total about $4 \cdot 10^{7}$ GTUs were recorded with the camera looking down on natural backgrounds like forests, lakes, and clouds, as well as city lights. Fig. 12 traces the time variation of a typical one of the 2304 pixels in the camera. The city of Timmins for example crossed the field of view of that pixel between 03:00 and 04:00 UTC. The low counts at the center of the data taking period correspond to times when the balloon was passing over forests, lakes, and clouds. The highest PE

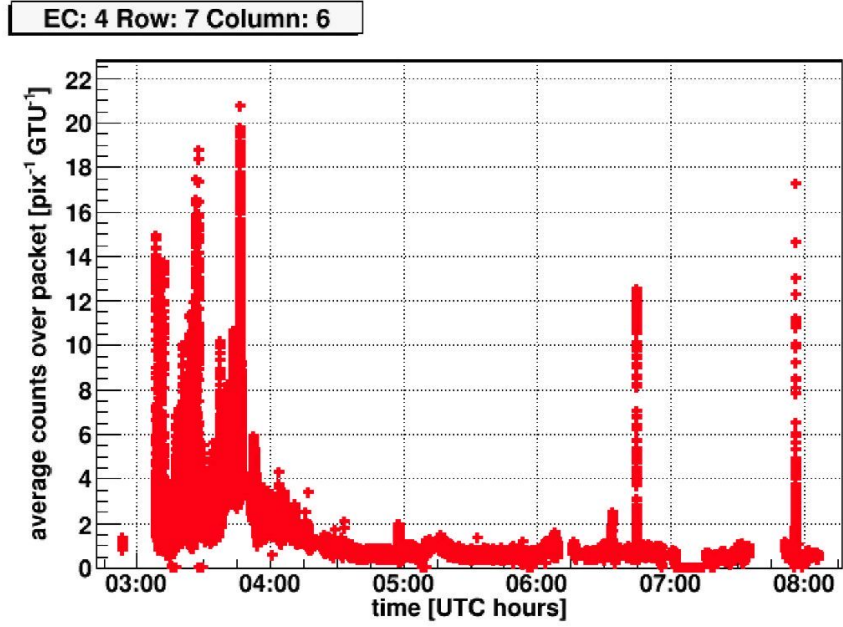

Fig. 12. PE counts averaged over each recorded data packet for one typical pixel, covering the entire balloon flight. 
counts were recorded when the balloon passed over an active mining operation.

For about two hours a helicopter was flying under the balloon. The helicopter carried three different light sources: a UV LED, a XeFlashlamp and a UV laser. This sources had the purpose of calibrating the detector response and simulating EAS-like event patterns that in this paper are used offline to test the FLT. Data analysis focused on about one hour of balloon flight during which the helicopter was shooting underneath the balloon and in the FoV of the camera. During this hour no other system tests interfered with the measurements, and the flight path crossed over dark as well as bright areas. This is essential to test the FLT logic under extreme conditions.

The data was divided into two data blocks: One block with so-called "nominal" background levels as are expected for operation on the ISS with $\sim 0.6 \mathrm{PE} / \mathrm{pixel} / \mathrm{GTU}$ averaged over a packet, and one block with a "high" background of 5-10 PE/pixel/GTU average over a packet in some parts of the FoV. This latter block of data was collected while passing over the mine or a city. About 15\% of MAPMT pixels that were not working properly were excluded from the analysis.

The light sources on the helicopter were set up to emit signature patterns that each served a distinct purpose [22]. First in the sequence was a UV-LED (375 $\mathrm{nm}$ wavelength), the light output of which steadily increased with time over 12 GTUs. From the balloon this signal appears as a stationary source typically contained in a single MAPMT pixel. The UV-LED light output was kept stable throughout the night and designed to raise the signal level from $\sim 1$ to $\sim 50$ PE over the 12 GTUs in that pixel. This signal provides a normalization for the distance between helicopter and EUSO-Balloon and allows to determine an effective threshold for the FLT.

Next in the sequence was a laser pulse shot horizontally away from the helicopter. This laser shot was fired about 25 GTU after the end of the UV-LED signal, delivering $\sim 5 \mathrm{~mJ}$ over $7.5 \mathrm{~ns}$ at a wavelength of 355 $\mathrm{nm}$. Depending on where in the balloon's FoV the helicopter happened to be at that time, it could take a maximum of 10 GTUs before the laser pulse would leave the balloon's FoV. The number of photons scattered out of such a laser pulse roughly corresponds to the fluorescence light emitted at shower maximum from a $\sim 10^{20} \mathrm{eV}$ EAS according to ESAF simulations [23].

The balloon's altitude being low compared to the ISS however meant that the $\sim 400 \mathrm{~m} \times 400 \mathrm{~m}$ of a $3 \times 3$ pixel cell on the ground was crossed by the laser pulse in $\sim 1 \mathrm{GTU}$, while the FLT is integrating over 5 GTU to establish a threshold crossing. To retain the ability for the FLT to trigger, the last light source in the sequence of light sources operated on the helicopter is a xenon (Xe) discharge lamp (wavelength $337 \mathrm{~nm}$ ) emitting its light over $\sim 8$ GTU, hereafter referred to as Xe flasher. This Xe flasher is triggered $\sim 5 \mu$ s after the laser shot, and its light curve reaches its maximum three GTU from its start, decreasing thereafter. This is reminiscent of the light curve along EAS, and four different flash intensities were used to mimic different EAS energies. A total helicopter light sequence therefore extends over $\sim \mathbf{5 0}$ GTU.

Fig. 13 shows the integrated PE count per pixel for a 128 GTU data packet containing a whole helicopter light sequence. An offset was subtracted throughout to highlight the excess along the laser track. The helicopter with the UV-LED and the Xe flasher was in the pixel at $x=5$, $y=25$. The left panel of Fig. 14 shows the PE sum for each GTU in that same data packet for the $3 \times 3$ pixel cell that is centered on the helicopter position at $x=5, y=25$. The UV-LED ramp can be seen to start from GTU 19. The laser shot is seen at GTU 55 and 56, and the Xe flash lamp is seen between GTU 58 and 65 . The signal peaking at GTU 72 is attributed to an afterpulse in the Xe flasher.

Fig. 13 also identifies the ECs that the various signals appear in. For the UV-LED and the Xe flasher this is EC number 4, and the laser track starts in EC number 4 and then moves out through ECs 5 and 6 . The right hand panel of Fig. 14 shows the number of ensuing FLT triggers per GTU for all ECs in that same data packet. The red line refers to triggers in EC number 4 , green to EC number 5, and blue to EC number 6 . All signals

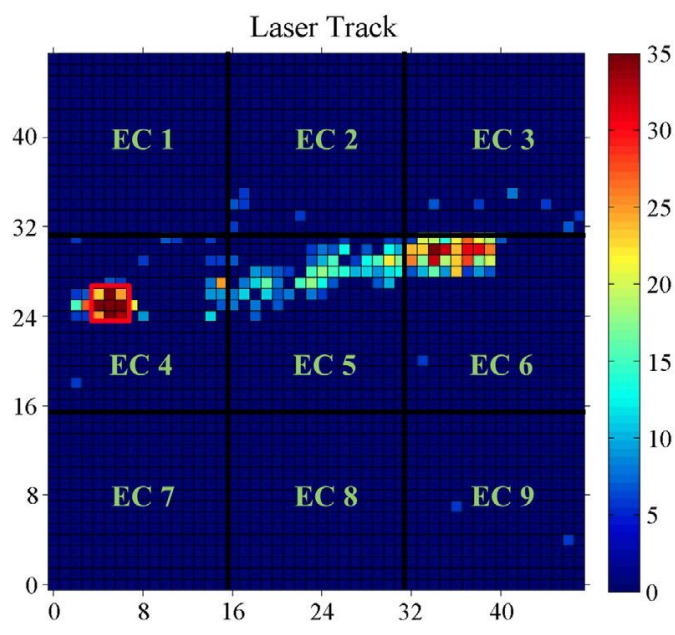

Fig. 13. Image of a helicopter event obtained by integrating the counts in each pixel for the whole packet $=1960$ of RUN $=043202$ (128 GTUs). A threshold is applied to the minimum signal level to emphasize the location of the track. The UV-LED and Xe-flasher signals are centered around pixel at axis of abscissae $X=5$ and axis of ordinates $Y=25$.

are detected by the FLT algorithm, and the delay between the signal and trigger timings simply reflect the 5 GTU integration.

As the EUSO-Balloon DAQ and the helicopter light sequence each run on their own respective clocks and the DAQ recorded only $2.5 \times 128$ $=320 \mu \mathrm{s}$ every $\sim 55 \mathrm{~ms}(\sim 0.6 \%)$, the vast majority of helicopter light sequences were not recorded. There will also be events where only a part of the helicopter light sequence overlapped with a DAQ data packet. Running the offline FLT simulation through the data, 274 events were found in which at least two ECs triggered the FLT algorithm.

Another peculiarity of EUSO-Balloon was that the optical module under the balloon spun, constantly changing the FoV's alignment with respect to both surface features and laser direction, and did so at a varying rate. This meant that especially at the edges of the FoV stationary and other light sources would often enter or exit the FoV during the $\sim 55 \mathrm{~ms}$ dead time between data packets. This complication should clearly be more relevant where stationary light sources on the ground play a significant role. As described above, the data was sorted into two blocks: one with nominal and the other with high background over locations lit up by human activity. The first block contains a total integrated time corresponding to $\sim 8.5 \mathrm{~s}$, and the latter corresponding to $\sim 6.5 \mathrm{~s}$, with about one order of magnitude more background light in this latter block's data on some parts of the FoV.

As expected the trigger rate under the more severe background conditions is higher: In the high background block of data the FLT algorithm triggered on 148 laser events and 59 others, while it triggered 126 laser and 17 other events in the nominal background data block. Assuming all other events are background, this puts the background rates for the current FLT trigger logic at $2.0 \mathrm{~Hz}$ per $9 \mathrm{ECs}$ for the nominal background data block and $9.1 \mathrm{~Hz}$ per 9 ECs for the high background data block. Under both conditions the rate requirement of $\sim 1 \mathrm{~Hz}$ per $\mathrm{EC}$ is met. In particular this means that despite the particular challenge posed by the combination of balloon spinning and DAQ deadtime the background estimation using the preceding data packet works well.

The event shown in Figs. 13 and 14 and triggered on by the FLT algorithm can be used to estimate an energy threshold for EAS that would pass the FLT. Averaging over the seven lowest PE/GTU values that raise trigger alerts in EC number 4 the average signal excess becomes $81 \pm 13$ and the average background $39 \pm 1 \mathrm{PE} / \mathrm{GTU}$. This is a signal over background ratio of $2.1 \pm 0.3$ for the $3 \times 3$ pixel cell raising the trigger. Comparing this to ESAF simulations for EUSO-Balloon [23] under nominal background conditions this ratio is reached for vertical EAS initiated by a $\sim 5 \times 10^{18} \mathrm{eV}$ proton. As the simulation also shows that showers at higher zenith angle provide higher signal/GTU, this 

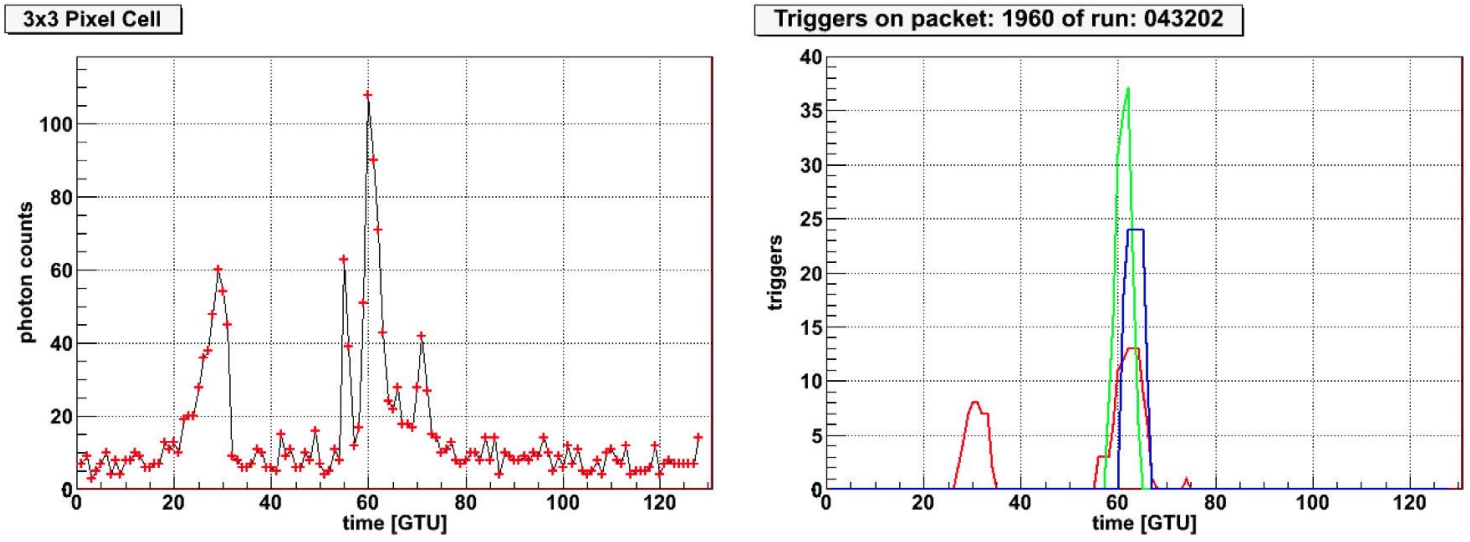

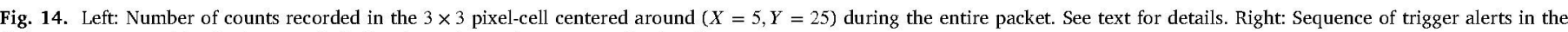
different ECs crossed by the laser track during the entire packet. See text for details.

value should be considered an upper limit for the energy threshold of FLT-triggered events recorded by EUSO-Balloon. Given its FoV and the measured CR rate at this energy this means that the FLT should trigger one event for every $24 \mathrm{~h}$ of EUSO-Balloon livetime.

\subsection{Tests with EUSO-TA data}

TurLab measurements and EUSO-Balloon data were used to verify that the FLT and in particular its background estimation perform and meet the requirements under various realistic or even challenging background conditions. While comparing the lowest light level in EUSOBalloon events that raised a FLT with simulation produced an estimate for the energy threshold in detecting cosmic ray particles, this is still a far cry from obtaining an efficiency curve for the FLT. This problem is addressed with data from EUSO-TA.

The EUSO-TA [18] telescope is a prototype of the JEM-EUSO space telescope with two $1 \mathrm{~m}^{2}$ square Fresnel lenses. Just as for EUSO-Balloon its electronics comprise a full PDM with 9 ECs and 36 MAPMTs.

It is located right in front of the Black Rock Mesa (BRM) fluorescence detector (FD) site of the TA experiment in the Utah West Desert, USA [24]. EUSO-TA's FoV of $11^{\circ} \times 11^{\circ}$ is contained within that of the BRM's FD and aligned such that it contains the vertical tracks from the pulsed $355 \mathrm{~nm}$ laser at TA's central laser facility (CLF). During TA data taking on the moonless parts of nights with amenable weather the CLF fires 300 vertical laser pulses of $3 \mathrm{~mJ}$ at $10 \mathrm{~Hz}$ every half hour. Providing atmospheric and calibration data for all three of TA's FD sites it is located centrally at an equal distance of $21 \mathrm{~km}$ from each of the TA FDs, and therewith also $21 \mathrm{~km}$ from EUSO-TA. Depending on the offset between GTU boundaries and laser shot, laser tracks took 6 to 8 GTUs for their image to cross the PDM at EUSO-TA. The left panel of Fig. 15 shows an average over $\sim 250$ such CLF shots as recorded by EUSO-TA.

The inclined laser track shown in the right panel of Fig. 15 is from a set of laser events produced with the help of a mobile UV laser belonging to the Colorado School of Mines. The missing piece in this laser track average was due to a defective MAPMT in EUSO-TA.

Also using a $355 \mathrm{~nm}$ laser the pulses from this mobile laser can be adjusted in intensity within a range of 1 to $86 \mathrm{~mJ}$. As the laser itself is steerable, the geometry of the laser track can be varied more freely, and for the average over the $\sim \mathbf{1 5 0}$ laser pulses shown here the laser was shot at a distance of $40 \mathrm{~km}$ with a pulse energy of $62 \mathrm{~mJ}$.

Varying the laser pulse energies with this mobile laser at $34 \mathrm{~km}$ from EUSO-TA produced the trigger efficiency curve for the FLT that is shown in Fig. 16. As at these distances the laser pulses typically cross a few pixel/GTU, the FLT logic was adapted by setting $N_{p s t}=1$, while $n_{\mathrm{thr}}^{\mathrm{pix}}$ and $n_{\mathrm{thr}}^{\text {cell }}$ were modified accordingly to keep the FLT trigger rates below the $1 \mathrm{~Hz} / \mathrm{EC}$ requirement. To determine the trigger efficiency, an external trigger, synchronized with the laser shooting, was supplied by

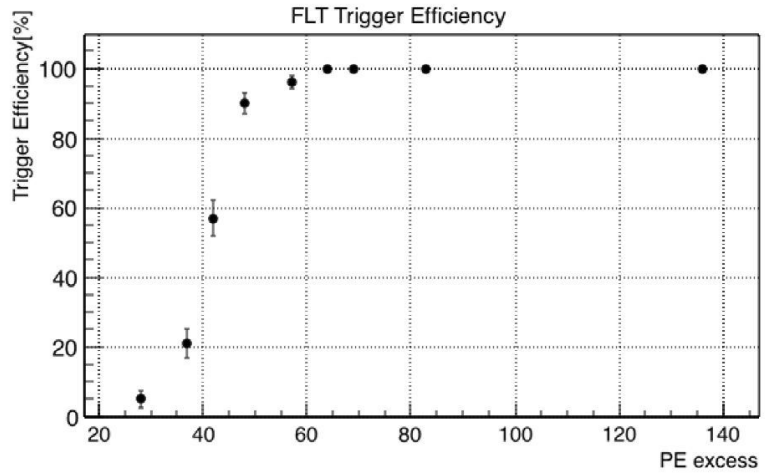

Fig. 16. Trigger efficiency curve of FLT as a function of the signal excess recorded by TA-EUSO.

the TA-FD to the EUSO-TA DAQ to always have the laser track inside a 128 GTU packet. The efficiency can then be determined by running the adapted FLT algorithm over these data packets and counting the packets that raise an FLT. Laser pulse energies between 3 and $5 \mathrm{~mJ}$ were used for the first four points with signal excesses above background $<50 \mathrm{PE}$ over all pixels in that GTU. Above 50 PE overall signal excess, which corresponds to $\sim 25 \mathrm{PE}$ in the relevant $3 \times 3$ pixel cell and $6 \mathrm{~mJ}$ pulse energy for this geometry, the trigger efficiency becomes $90 \%$ and higher. In a corresponding analysis for the $21 \mathrm{~km}$ CLF geometry the CLF's $3 \mathrm{~mJ}$ pulses were seen with $94 \%$ efficiency.

\section{Conclusions}

The FLT logic for use in JEM-EUSO as described above and implemented in VHDL was shown here (a) to work well in the presence of artificially produced as well as naturally encountered fluctuations in the background lighting conditions and (b) to keep the FLT rate within the permissible bounds while (c) being efficient at identifying event types with general EAS characteristics.

The FLT trigger as presented here is working at the MAPMT level and is based on the local persistency of a signal excess in a $3 \times 3$ pixel area, persisting a few GTUs. To achieve this an automatic evaluation of the average background level is derived from the preceding data package, as strategy that has proven successful even when individual data packages were separated by up to a few hundred $\mu$ s. Rejection for events with time duration too large for an EAS signal, namely longer than 72 GTUs on the ISS, is also implemented. This implementation for one EC requires only a few per cent of the resources of commercial FPGAs, which allows to implement it within the power constraints imposed on the ISS. 

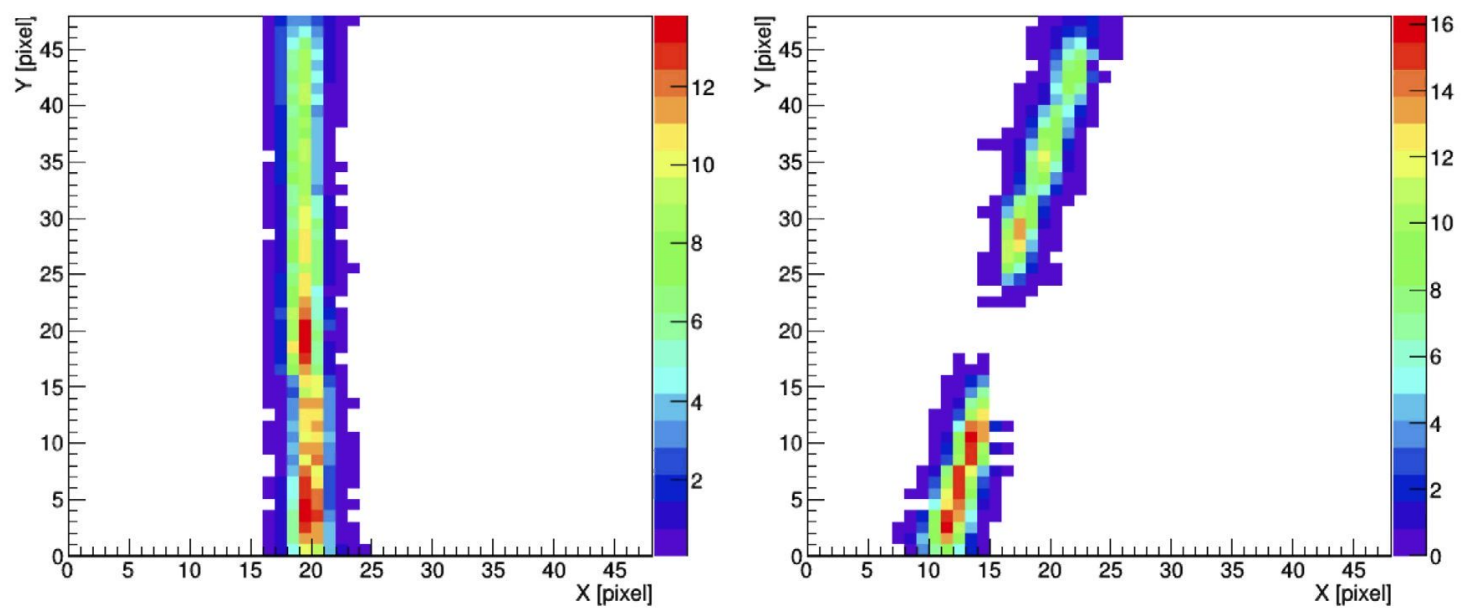

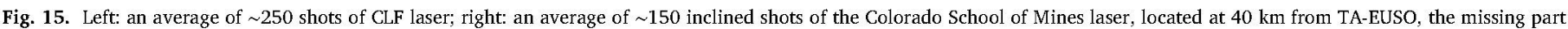

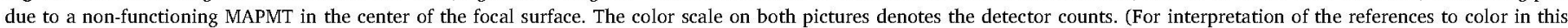
figure legend, the reader is referred to the web version of this article.)

Source: Figure taken from [25].

Tests performed with EUSO-Balloon and EUSO-TA data, as well as measurements performed at the TurLab facility, allowed validating the main functions of the algorithm. The system automatically adjusts the thresholds to keep the rate of triggers on background fluctuations below $1 \mathrm{~Hz} / \mathrm{EC}$ even in the case of slow background variations. The FLT level trigger detects EAS-like events with light intensities comparable to those JEM-EUSO would observe in the energy range $E>5 \cdot 10^{19}$ $\mathrm{eV}$ and in the presence of expected night sky background. These results strengthen those obtained in [7] and successive publications as they show that the trigger concept developed from simulation can be effectively implemented in hardware and performs well on real data.

The FLT has shown to be quite effective in rejecting city-like and other slow but bright events such as meteors. Of the few spurious triggers that occurred most were artifacts of discontinuities introduced by the available equipment.

The examples shown in this paper are only a sub-sample of all tests performed on the data reported here and the ongoing activities at the TurLab facility and EUSO-TA.

The VHDL logic of the FLT is currently being implemented on the FPGA of the PDM board. EUSO-SPB [26], the next stratospheric balloon flight, is expected to host this trigger logic on-board to verify its performance on real EAS.

\section{Acknowledgments}

This work was partially supported by the Basic Science Interdisciplinary Research Projects of RIKEN and JSPS KAKENHI Grant (22340063, 23340081, and 24244042), by the Italian Ministry of Foreign Affairs and International Cooperation, by the 'Helmholtz Alliance for Astroparticle Physics HAP' funded by the Initiative and Networking Fund of the Helmholtz Association, Germany, and by Slovak Academy of Sciences MVTS JEM-EUSO as well as VEGA grant agency project $2 / 0076 / 13$. Russia is supported by the Russian Foundation for Basic Research Grant No. 13-02-12175-ofi-m. The Spanish Consortium involved in the JEM-EUSO Space Mission is funded by MICINN \& MINECO under the Space Program projects: AYA2009-06037-E/AYA, AYA-ESP2010-19082, AYAESP2011-29489-C03, AYA-ESP2012-39115-C03, AYA-ESP2013-47816C4, MINECO/FEDER-UNAH13-4E-2741, CSD2009-00064 (Consolider MULTIDARK) and by Comunidad de Madrid (CAM) under projects S2009/ESP-1496 \& S2013/ICE-2822. The activities at TurLab facility have been partially funded by the European High-Performance
Infrastructures in Turbulence (EuHIT). The authors acknowledge strong support from the French Space Agency CNES who provided besides funding - the leadership that made the EUSO-Balloon project possible in a very short time. We are deeply indebted to the balloon division of CNES for a perfect launch, smooth flight operation and flawless telemetry. The Canadian Space Agency has provided outstanding facilities at the Timmins Stratospheric Balloon Base, and a quick and careful recovery of the instrument. The authors dedicate this paper to the memory of Dr. Jacek Karczmarczyk and Dr. Yoshiya Kawasaki, who have contributed greatly to the project and will be deeply missed.

\section{References}

[1] R. Benson, J. Linsley, Proc. 17th Int. Cosmic Ray Conf. (Paris), 1981, p. 8.

[2] J.H. Adams, et al. (JEM-EUSO Coll.), Exp. Astron. 40 (2015) 3.

[3] F. Kajino, et al. (JEM-EUSO Coll.), Nucl. Instrum. Methods A 623 (2010) 422

[4] J.H. Adams, et al. (JEM-EUSO Coll.), Exp. Astron. 40 (2015) 19.

[5] M. Bertaina, et al. (JEM-Euso Coll.), Adv. Space Res. 53 (2014) 1515.

[6] C. Berat, et al., Astrop. Phys. 33 (2010) 221.

[7] J.H. Adams Jr., et al. (JEM-EUSO Coll.), Astropart. Phys. 44 (2013) 76.

[8] M. Sato, et al., Internat. J. Modern Phys. A 20/29 (2005) 6903.

[9] N. Sakaki et al., Proc. 30th Int. Cosmic Ray Conf. (Merida), HE 5, 2007, p. 1037.

[10] G.K. Garipov, et al. (Tatiana Coll.), JETP Lett. 4 (2005) 185.

[11] J. Bayer, et al., (JEM-EUSO Coll.), Proc. 32th Int. Cosmic Ray Conf. (Beijing), 3, 2011, p. 168; arXiv:12045065.

[12] J. Bayer, (JEM-EUSO Coll.), Proc. 33th Int. Cosmic Ray Conf. (Rio de Janeiro), \#0432, 2013; arXiv:13077071.

[13] M. Bertaina, et al. (JEM-EUSO Coll.), Nucl. Instrum. Methods A 824 (2016) 253.

[14] http://www.xilinx.com/support/documentation/data_sheets/ds150.pdf.

[15] M. Bertaina, et al. (JEM-EUSO Coll.), Exp. Astron. 40 (2015) 117.

[16] J.H. Adams, et al. (JEM-EUSO Coll.), Exp. Astron. 40 (2015) 281.

[17] M. Bertaina, et al. (JEM-EUSO Coll.), EPJ Web of Conferences 89 (2015) 03003.

[18] J.H. Adams, et al. (JEM-EUSO Coll.), Exp. Astron. 40 (2015) 301.

[19] http://www.arduino.cc.

[20] P. von Ballmoos, et al., (JEM-EUSO Coll.), Proc. 34th Int. Cosmic Ray Conf. (Den Haag), \#0725, 2015.

[21] M. Bertaina, et al., (JEM-EUSO Coll.), Proc. 34th Int. Cosmic Ray Conf. (Den Haag), \#0890, 2015.

[22] J. Eser, et al., (JEM-EUSO Coll.), Proc. 34th Int. Cosmic Ray Conf. (Den Haag), \#0860, 2015.

[23] F. Fenu, et al., (JEM-Euso Coll.), Proc. 34th Int. Cosmic Ray Conf. (Den Haag), \#0639, 2015.

[24] T. Abu-Zayyad, et al. (Telescope Array Coll.), Nucl. Instrum. Methods A 689 (2012) 87.

[25] M. Casolino, et al., (JEM-EUSO Coll.), Proc. 34th Int. Cosmic Ray Conf. (Den Haag), \#0636, 2015.

[26] L. Wiencke, et al., (JEM-EuSO Coll.), Proc. 34th Int. Cosmic Ray Conf. (Den Haag), \#0165, 2015. 